ARTICLE

https://doi.org/10.1038/s41467-019-10202-3

\title{
6mA-DNA-binding factor Jumu controls maternal- to-zygotic transition upstream of Zelda
}

\author{
Shunmin He (10) 1,2,8, Guoqiang Zhang (10 1,8, Jiajia Wang ${ }^{2,8}$, Yajie Gao ${ }^{1,3,8}$, Ruidi Sun ${ }^{1,4}$, Zhijie Cao1, \\ Zhenping Chen ${ }^{1}$, Xiudeng Zheng (10 5 , Jiao Yuan², Yuewan Luo ${ }^{1,3}$, Xiaona Wang ${ }^{1}$, Wenxin Zhang ${ }^{1,4}$, Peng Zhang ${ }^{2}$, \\ Yi Zhao ${ }^{6}$, Chuan He (i) ${ }^{7}$, Yi Tao (i) ${ }^{5}$, Qinmiao Sun ${ }^{1,3} \&$ Dahua Chen ${ }^{1,3}$
}

\begin{abstract}
A long-standing question in the field of embryogenesis is how the zygotic genome is precisely activated by maternal factors, allowing normal early embryonic development. We have previously shown that N6-methyladenine (6mA) DNA modification is highly dynamic in early Drosophila embryos and forms an epigenetic mark. However, little is known about how $6 \mathrm{~mA}$ formed epigenetic information is decoded. Here we report that the Fox-family protein Jumu binds $6 \mathrm{~mA}$-marked DNA and acts as a maternal factor to regulate the maternal-to-zygotic transition. We find that zelda encoding the pioneer factor Zelda is marked by $6 \mathrm{~mA}$. Our genetic assays suggest that Jumu controls the proper zygotic genome activation (ZGA) in early embryos, at least in part, by regulating zelda expression. Thus, our findings not only support that the $6 \mathrm{~mA}$-formed epigenetic marks can be read by specific transcription factors, but also uncover a mechanism by which the Jumu regulates ZGA partially through Zelda in early embryos.
\end{abstract}

\footnotetext{
${ }^{1}$ State Key Laboratory of Membrane Biology, Institute of Zoology, Chinese Academy of Sciences, Beijing 100101, China. ${ }^{2}$ Key Laboratory of RNA Biology, Center for Big Data Research in Health, Institute of Biophysics, Chinese Academy of Sciences, Beijing 100101, China. ${ }^{3}$ School of Life Sciences, University of Chinese Academy of Sciences, Beijing 100049, China. ${ }^{4}$ School of Life Sciences, Anhui Agricultural University, Hefei, Anhui 230036, China. ${ }^{5}$ Centre for Computational and Evolutionary Biology, Key Laboratory of Animal Ecology and Conservational Biology, Institute of Zoology, Chinese Academy of Sciences, Beijing 100101, China. ${ }^{6}$ Advanced Computer Research Center, Institute of Computing Technology, Chinese Academy of Sciences, Beijing 100190, China.

${ }^{7}$ Department of Chemistry and Institute for Biophysical Dynamics, Howard Hughes Medical Institute, The University of Chicago, Chicago, IL 60637, USA.

${ }^{8}$ These authors contributed equally: Shunmin He, Guoqiang Zhang, Jiajia Wang, Yajie Gao. Correspondence and requests for materials should be addressed to Y.T. (email: yitao@ioz.ac.cn) or to Q.S. (email: qinmiaosun@ioz.ac.cn) or to D.C. (email: chendh@ioz.ac.cn)
} 
M ethylated bases, such as 5-methylcytosine $(5 \mathrm{mC})$ and N6-methyladenine $(6 \mathrm{~mA})$, are the most abundant types of DNA modification in the genomes of diverse species $^{1,2} .5 \mathrm{mC}$ modification has been studied extensively in higher eukaryotes. Previous studies have suggested that the dynamic regulation of $5 \mathrm{mC}$ plays important roles in regulating chromatin architecture and gene expression during mammalian development and adult tissue homeostasis ${ }^{3-6}$. Contrary to the significant progresses made in understanding cytosine modification, the role of $6 \mathrm{~mA}$ was considered less important in higher eukaryotes ${ }^{7}$. Recent studies suggest that $6 \mathrm{~mA}$ associates with gene expression in multiple eukaryotic species, including algae, worm, fly, and mammals, indicating that $6 \mathrm{~mA}$ has potential epigenetic roles in eukaryotes ${ }^{8-13}$. However, little is known about mechanisms of how 6mA-related epigenetic codes are formed and interpreted to direct gene expression programs.

At the early embryonic stages, all animal fertilized embryos undergo a critical process, namely the maternal-to-zygotic transition (MZT). During MZT, a proportion of the oocyteloaded maternal products are cleared and the zygotic genome is activated. While maternal mRNA clearance is triggered by a number of RNA-binding proteins, zygotic genome activation (ZGA) is primarily instructed by a small number of pioneer transcription factors ${ }^{14-17}$. In Drosophila, early embryogenesis is characterized by a series of rapid synchronous nuclear divisions. The zygotic genome is almost quiescent in the first few rounds of nuclear division (before cycles 7 or 8$)^{16,18}$, and becomes widely activated when the MZT ends at the 14th cycle $^{19}$. It has been shown that Zygotic early Drosophila activator (Zelda) (or called Vfl) functions as a pioneer transcription factor and accesses the early embryonic genome by binding sequence-specific motifs (referred to as TAGteam sites), and subsequently increases chromatin accessibility for other transcription factors, thus ensuring coordinated gene expression during $\mathrm{MZT}^{20-24}$. Of note, the proper expression of Zelda is critical for early embryogenesis, since either loss of or overexpression of Zelda leads to defects of embryonic development ${ }^{20-22,24}$, raising a possibility that an uncharacterized mechanism coordinates with the pioneer factor Zelda to regulate early embryonic events.

We have previously shown that $6 \mathrm{~mA}$ is highly dynamic in Drosophila early embryos. Notably, the timing window of the $6 \mathrm{~mA}$ dynamics almost corresponded to the MZT process during early embryogenesis ${ }^{17,23,25}$. We speculate that $6 \mathrm{~mA}$ may contribute to MZT by forming an epigenetic code that can be recognized by maternal factors in early embryos. In this study, we show that the Fox family protein Jumu functions as a maternal transcription factor and regulates embryonic gene expression by preferentially binding the $6 \mathrm{~mA}$-marked DNA. Importantly, we find that zelda is marked with $6 \mathrm{~mA}$ and regulated by Jumu. Our genetic analyses show that partial knockdown of Zelda significantly suppresses the embryonic lethal phenotype induced by loss of maternal Jumu. Together, our findings suggest that Jumu preferentially binds 6mA-marked DNA and controls MZT, at least in part through regulating Zelda.

\section{Results}

Landscape of $6 \mathrm{~mA}$ modification in early embryonic genomes. To explore the potential role of $6 \mathrm{~mA}$ in MZT, we first characterized the genome-wide features of $6 \mathrm{~mA}$. We collected genomic DNA from 0.75 -h (nearly pre-MZT and pre-ZGA), 3-h (post-ZGA), and 6-h (post-MZT) post-fertilization embryos (see Methods; Fig. 1a) and then employed an anti-6mA antibody (Abcam) to perform DNA immunoprecipitation (DNA-IP) experiments (see Methods; Supplementary Figure 1a-c). The IPed
DNAs were then subjected to the paired-end (125 bp) highthroughput sequencing (Supplementary Figure 1d).

We identified $17,5286 \mathrm{~mA}$ peaks at the 0.75 -h stage and several thousands of peaks at the 3 -h and 6-h stages (Fig. $1 \mathrm{~b}$ and Supplementary Figure 1e, f). The signal strength of the $6 \mathrm{~mA}$ peaks in the 0.75 - $h$ sample was much stronger than that in the two later stages (Fig. 1c and Supplementary Figure 1g). Moreover, about $80 \%(13,897 / 17,528)$ of $6 \mathrm{~mA}$ peaks identified at the $0.75-\mathrm{h}$ stage had disappeared at the 3 -h stage, although these peak regions still retained faint $6 \mathrm{~mA}$ signals in the 3 -h samples (Fig. 1b, d and Supplementary Figure 1e). Additionally, about $20 \%$ of peaks identified at the 0.75 -h stage were also present at the 3 -h stage, but $6 \mathrm{~mA}$ signals in these common peaks were weaker at the 3 -h stage (Fig. 1d), suggesting that $6 \mathrm{~mA}$ modification were dynamically regulated during early embryogenesis (Fig. 1e).

We have previously shown that DMAD, a $6 \mathrm{~mA}$ demethylase in Drosophila, is essential for $6 \mathrm{~mA}$ demethylation and embryogenesis. To investigate whether DMAD is involved in regulating the $6 \mathrm{~mA}$ landscape in early embryo genomes, we collected genomic DNA from DMAD knockdown embryos (Supplementary Figure $1 \mathrm{~h}$ ) at the stage of 6 -h post fertilization, and performed DNAIP followed by paired-end high-throughput sequencing. In contrast to the control (6-h wild-type embryos), from which we obtained 2,447 $6 \mathrm{~mA}$ peaks, the number of $6 \mathrm{~mA}$ peaks from $D M A D$ knockdown embryos was increased to 8,367 (Fig. If and Supplementary Figure 1i). These peaks were highly overlapped with those in 0.75-, 3- and 6-h wild-type samples (Fig. 1f). Moreover, when compared with the 6-h wild-type samples, approximately $79 \%$ of the gained $6 \mathrm{~mA}$ peaks in $6-\mathrm{h}$ DMAD knockdown embryos were also found in the 0.75 -h or 3-h samples (Fig. 1f and Supplementary Figure 1i) and the signal of these gained $6 \mathrm{~mA}$ peaks also exhibited a dynamic pattern from $0.75-\mathrm{h}$ to 6-h (Fig. 1g, h), suggesting that DMAD plays an important role in controlling dynamics of $6 \mathrm{~mA}$ modification in early embryonic genomes.

$6 \mathrm{~mA}$ can mark coding genes in early embryos. We have previously reported that $6 \mathrm{~mA}$ marks transposon sequences in ovaries. Consistent with this observation, we found that, in addition to transposons, such as LTR and LINE, a portion of $6 \mathrm{~mA}$ peaks in early embryo was enriched with simple repeat regions (Supplementary Figure 2a, b). As shown in Fig. 2a, about 33 43\% of $6 \mathrm{~mA}$ peaks were mapped to the intronic regions, and $36 \sim 45 \%$ of $6 \mathrm{~mA}$ peaks were found to locate in intergenic regions at different embryonic stages. Further analyses revealed that $49-72 \%$ of intronic $6 \mathrm{~mA}$ peaks and $63-74 \%$ of intergenic $6 \mathrm{~mA}$ peaks were located in transposons and simple repeat regions (Fig. 2a). Of note, although a large proportion of $6 \mathrm{~mA}$ peaks were mapped to the simple repeat and transposons, many of these peaks were also found to locate nearby coding genes or within the introns, and were considered as coding gene-related $6 \mathrm{~mA}$ peaks in this study. In total, we identified $54-62 \%$ of coding gene-related $6 \mathrm{~mA}$ peaks (see Methods; Fig. 2a).

To test whether $6 \mathrm{~mA}$ modification is associated with the coding gene expression in embryos, we performed RNA-seq with embryo samples at 0.75-, 3-, and 6-h stages and found 6,723 differentially expressed genes between at least two different stages. These genes were then clustered into several groups (Supplementary Figure 2c). Based on their expression level trends, we defined group 1 (489 genes) as a typical group of zygotically activated genes (Supplementary Figure $2 c, d$ ), and found that $6 \mathrm{~mA}$-marked genes were significantly enriched $(P=5.34 \mathrm{e}-28)$ in zygotically activated genes (Fig. 2b).

We next tested whether $6 \mathrm{~mA}$ influences coding gene and transposon expression in embryos. By analyzing the RNA-seq 
a

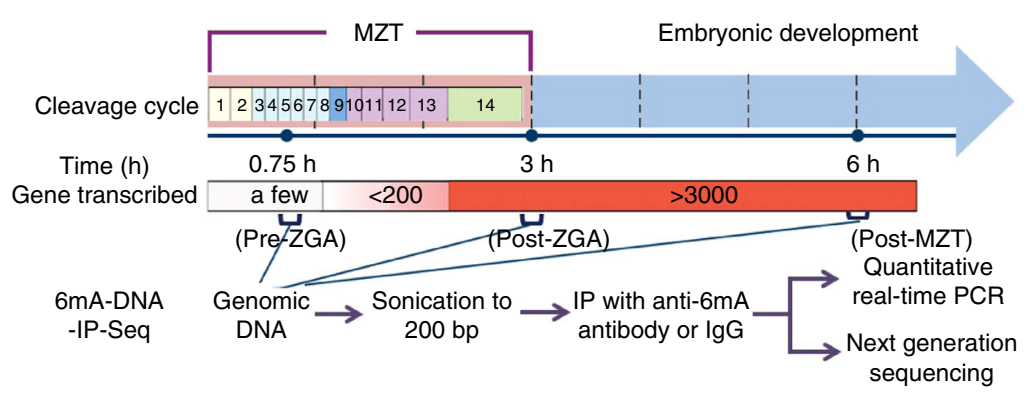

b

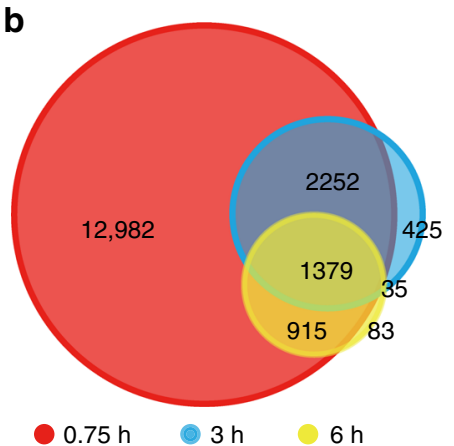

C

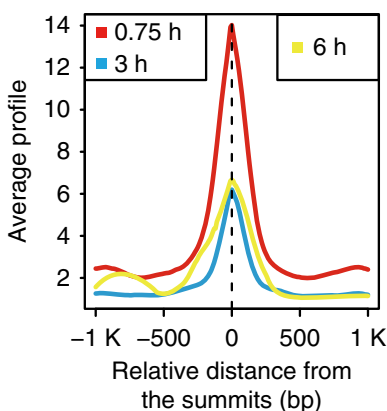

d
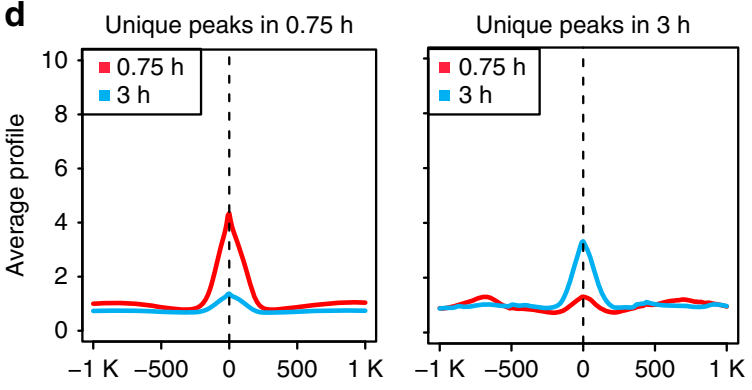

Common peaks in $0.75 \mathrm{~h} / 3 \mathrm{~h}$

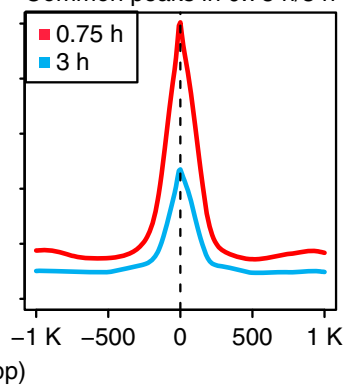

e
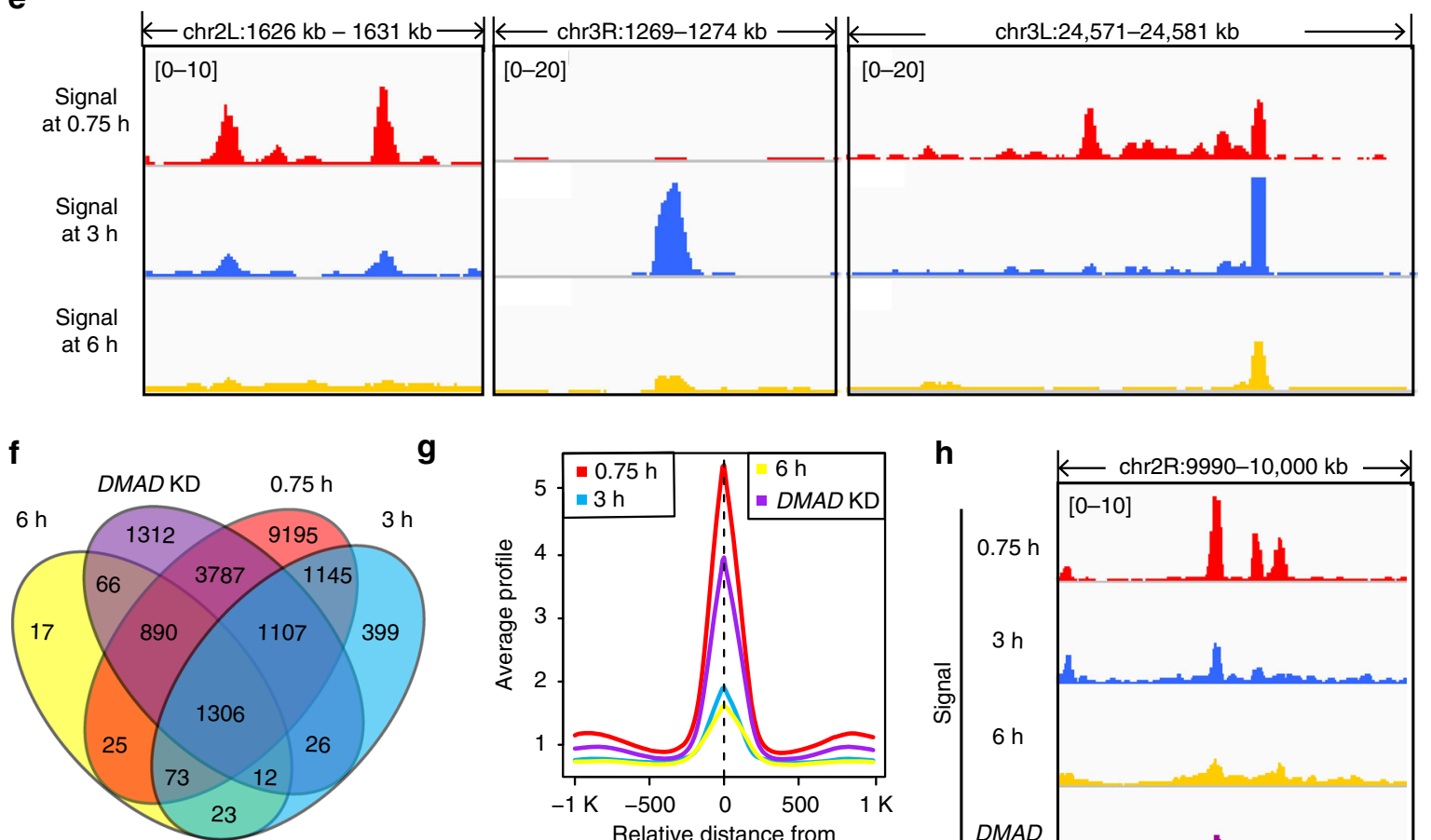

g

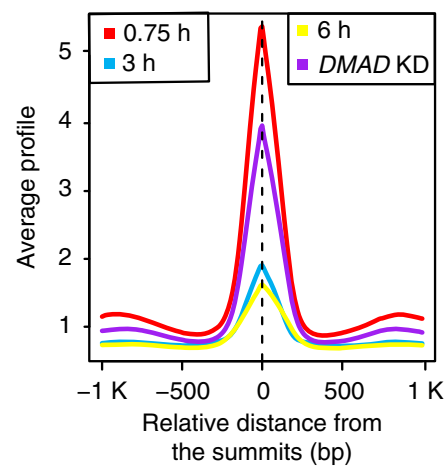

h

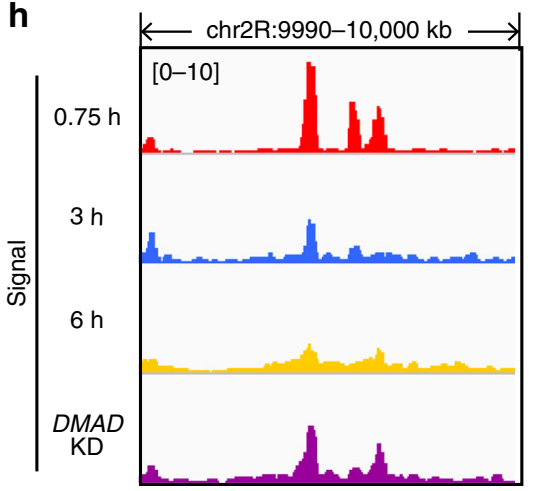

Fig. 1 Dynamic distribution of $6 \mathrm{~mA}$ in Drosophila early embryo genomes. a Timeline of the early embryogenesis and the 6mA-DNA-IP-Seq experimental process. The cleavage cycle is described according to a previous study ${ }^{25}$. DNA samples were collected from 0.75-h (nearly pre-MZT and pre-ZGA), 3-h (post-ZGA), and 6-h (post-MZT) post-fertilization embryos. b Overlap of 6mA enrichment peaks in 0.75-, 3-, and 6-h stage embryos. In cases where one peak in one sample overlapped multiple peaks in another sample, we selected the overlapped peak number from one sample as representative. c The average $6 \mathrm{~mA}$ signal profiles for the common peaks in $0.75-, 3-$, and 6-h stage embryos. $\mathbf{d}$ The average $6 \mathrm{~mA}$ signal profiles in 0.75- and 3-h unique peaks and their common peaks. e Examples of $6 \mathrm{~mA}$-marked regions in which the $6 \mathrm{~mA}$ modification signals were dynamically changed in early embryos. $\mathrm{f}$ Overlap of $6 \mathrm{~mA}$ peaks identified in 0.75-, 3- and 6-h of wild type and 6-h DMAD knockdown (KD) samples. $\mathbf{g}$ The average 6mA signal profiles in peaks gained in 6h DMAD knockdown samples, when compared with 6-h wild-type samples. These peaks were also detected in samples at 0.75-h or 3-h stages. $\mathbf{h}$ An example of 6mA signal in 0.75-, 3- and 6-h of wild-type stage and 6-h DMAD knockdown samples. MZT maternal-to-zygotic transition, ZGA zygotic genome activation 

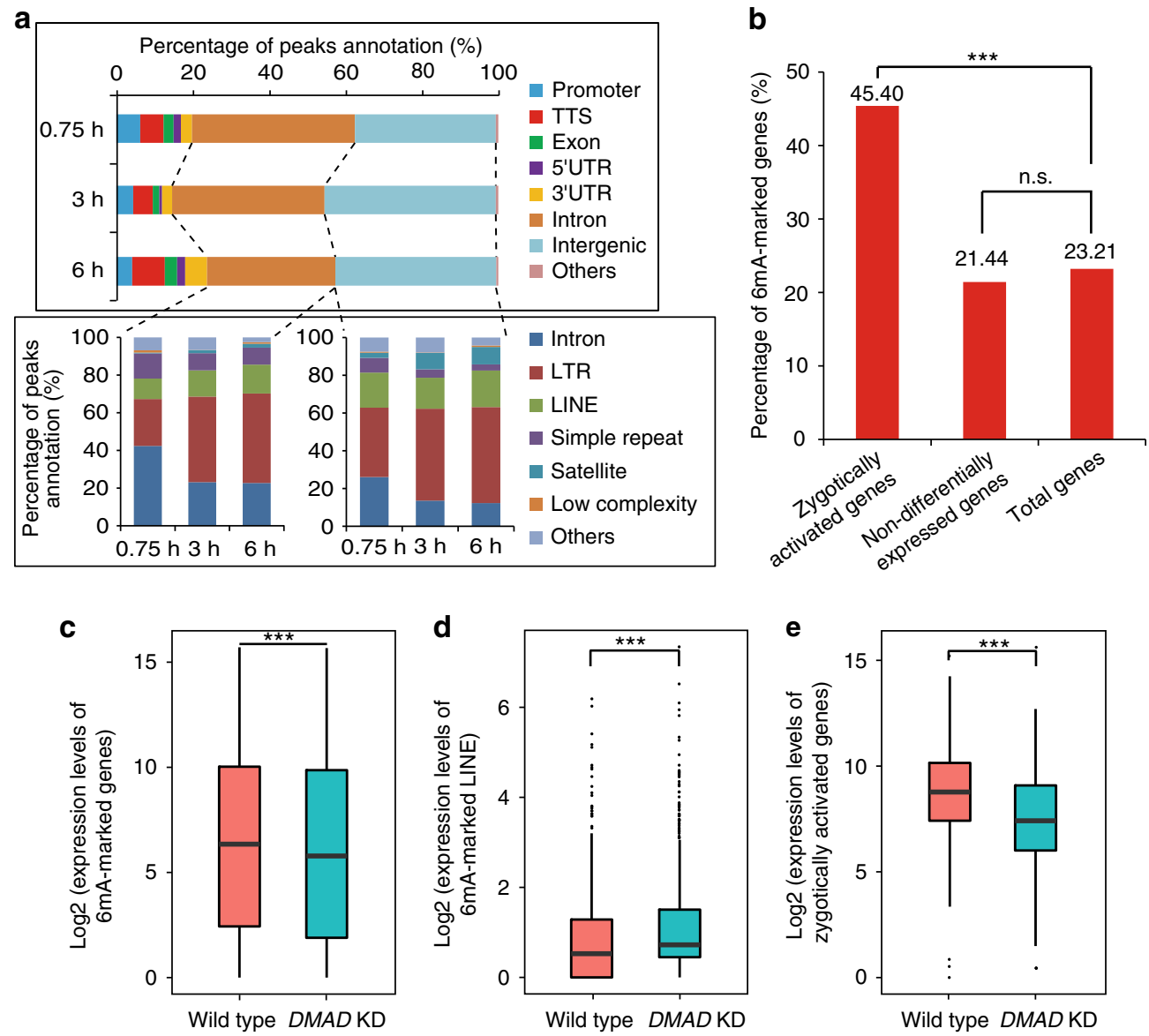

Fig. $26 \mathrm{~mA}$ marks coding genes and associates with gene expression in early embryos. a Distribution of $6 \mathrm{~mA}$ peaks on the Drosophila genome. Promoter, from $1 \mathrm{~kb}$ upstream to $100 \mathrm{bp}$ downstream of the TSS of a gene; TTS, from $100 \mathrm{bp}$ upstream to $1 \mathrm{~kb}$ downstream of transcription terminal site of a gene; Intergenic, $1 \mathrm{~kb}$ away from any gene. LTR and LINE are transposons. b Percentage of genes marked with $6 \mathrm{~mA}$. $P$ value was calculated by one-tailed hypergeometric test. ${ }^{\star \star \star} P<0.001$. c-e The expression of $6 \mathrm{~mA}$-marked genes (c), $6 \mathrm{~mA}$-marked LINEs (d), zygotically activated genes (e) in wild type and DMAD knockdown (KD) embryos. $P$ values were calculated by one-tailed Student's $t$ test. ${ }^{\star \star \star} P<0.001$. For both boxplots, the centre line indicates the median, the bottom and top of the box show the first and third quartiles of the data, and the whiskers show the minimum and maximum values. Source data are provided as a Source Data file. TSS transcription start site, TTS transcription terminal site, n.S., not significant

data from $D M A D$ knockdown and wild-type samples at the 6-h stage, we found that while the expression of LINE transposons was significantly up-regulated, the expression level of total $6 \mathrm{~mA}-$ related coding genes was significantly downregulated in $D M A D$ knockdown samples (Fig. 2c, d and Supplementary Figure 2e, f). Particularly, we noted that the expression of zygotically activated genes was significantly downregulated in DMAD knockdown sample (Fig. 2e and Supplementary Figure 2g). Thus, our findings suggest that $6 \mathrm{~mA}$ modification associates with gene expression in early embryos.

Identification of Jumu as a $6 \mathrm{~mA}$-DNA-binding protein. To identify potential $6 \mathrm{~mA}-\mathrm{DNA}$-binding proteins, we analyzed the DNA sequences of $6 \mathrm{~mA}$ peak regions, and found that Forkhead domain (FHD)-binding motifs were highly enriched in the $6 \mathrm{~mA}$ peak regions (Fig. 3a), raising a possibility that a Fox family protein binds to the $6 \mathrm{~mA}-\mathrm{DNA}$ and acts as a maternal factor to control early embryogenesis. We therefore performed affinity purification experiments using $6 \mathrm{~mA}$-modified DNA probes. Based on our $6 \mathrm{~mA}$ data sets, we synthesized biotin-labeled DNA probes with or without $6 \mathrm{~mA}$ modification and mixed probes with nuclear lysate from early embryos. Complexes immunoprecipitated by the biotin-labeled DNA probes were subjected to mass spectrometry analyses. Intriguingly, we found that Jumu, a Fox family protein, was enriched in the immunoprecipitants purified by the $6 \mathrm{~mA}$-modified probes (Supplementary Figure 3a). We analyzed the expression level of all Fox family genes at $0.75-, 3$ and 6-h, and found that jumu displayed the highest expression levels at all three stages (Supplementary Figure 3b). To test whether Jumu is a 6mA-DNA-binding protein, we performed western blot assays using an anti-Jumu antibody against purified complexes. As shown in Fig. 3b, complexes purified by the $6 \mathrm{~mA}-$ modified probes exhibited a much higher Jumu signal than the control. We then performed gel-shift assays to measure the binding affinity of Jumu with 6mA-modified or unmodified DNA probes (FAM-labeled) that contained the FHD-binding sequence. The probe competition analysis revealed that Jumu preferentially bound the $6 \mathrm{~mA}$-modified DNA probe (Fig. 3c-e). To obtain further evidence, we measured the binding affinity of Jumu with $6 \mathrm{~mA}$-modified or unmodified probes by performing the surface plasmon resonance (SPR) kinetic assays, and found Jumu had much higher binding activity for $6 \mathrm{~mA}-\mathrm{DNA}(K \mathrm{~d}=2.37 \mu \mathrm{M})$ than for unmodified DNA $(K \mathrm{~d}=42.1 \mu \mathrm{M})$ (Fig. 3f). Together, our findings suggest that Jumu preferentially binds the $6 \mathrm{~mA}$-modified DNA probes.

To rule out the preferential binding of Jumu with $6 \mathrm{~mA}$ modified DNA was attributed to the sequence specificity of the probes, we incubated the purified Flag-Jumu protein or the FlagGFP protein (used as control) with genomic DNA from wild type 
a

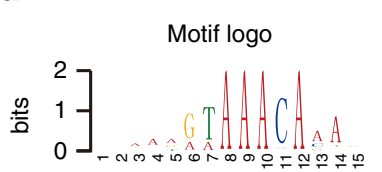

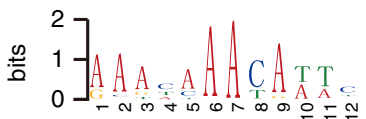

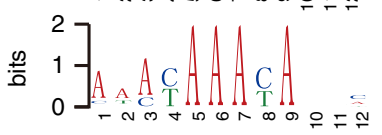

C

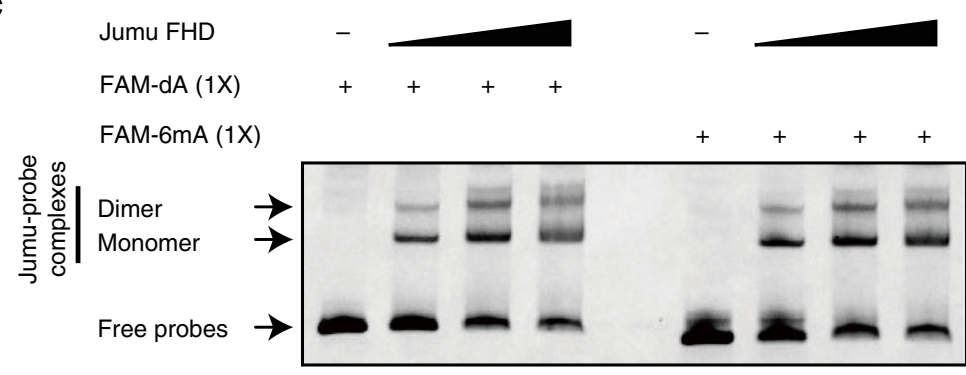

d

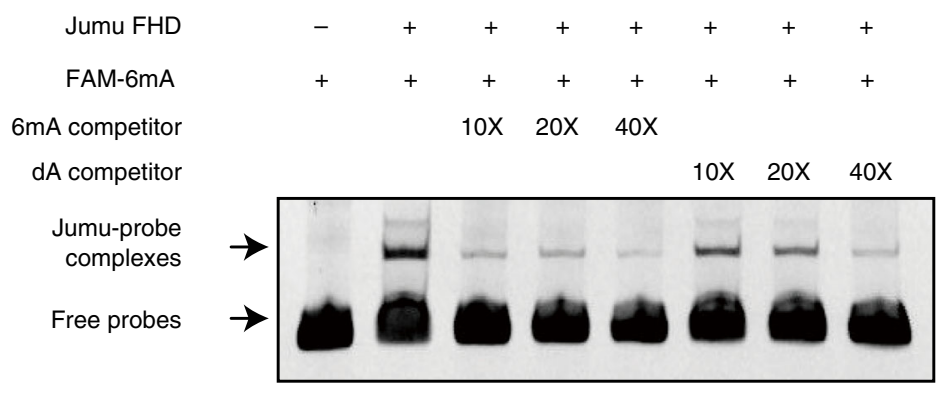

e

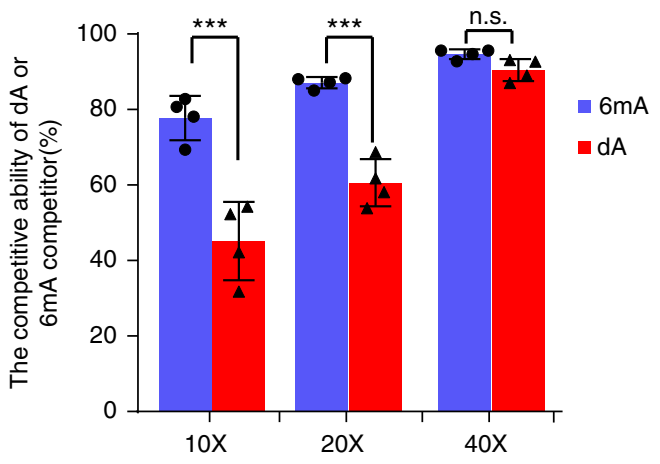

g

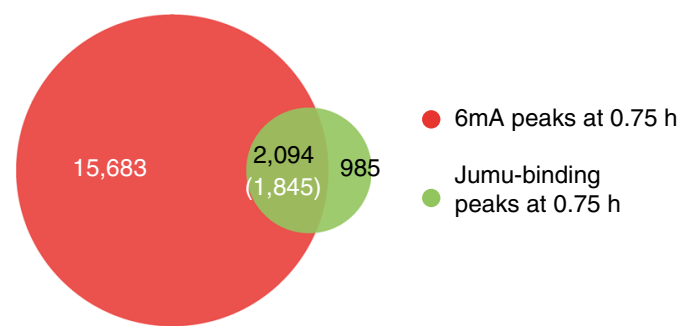

$0.75 \mathrm{~h}$

No. of peaks(\%)

$6284(35.85 \%)$

6.7E-54

2. $2 \mathrm{E}-18$

$5949(33.94 \%)$

2.0E-118

FOXP1

FOXD3

$(59.30 \%)$
$6251(35.66 \%)$

h b

(\%)

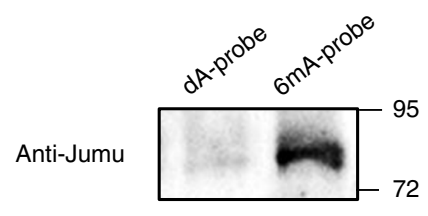

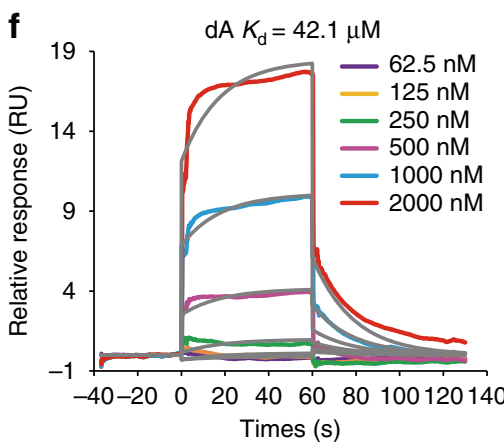
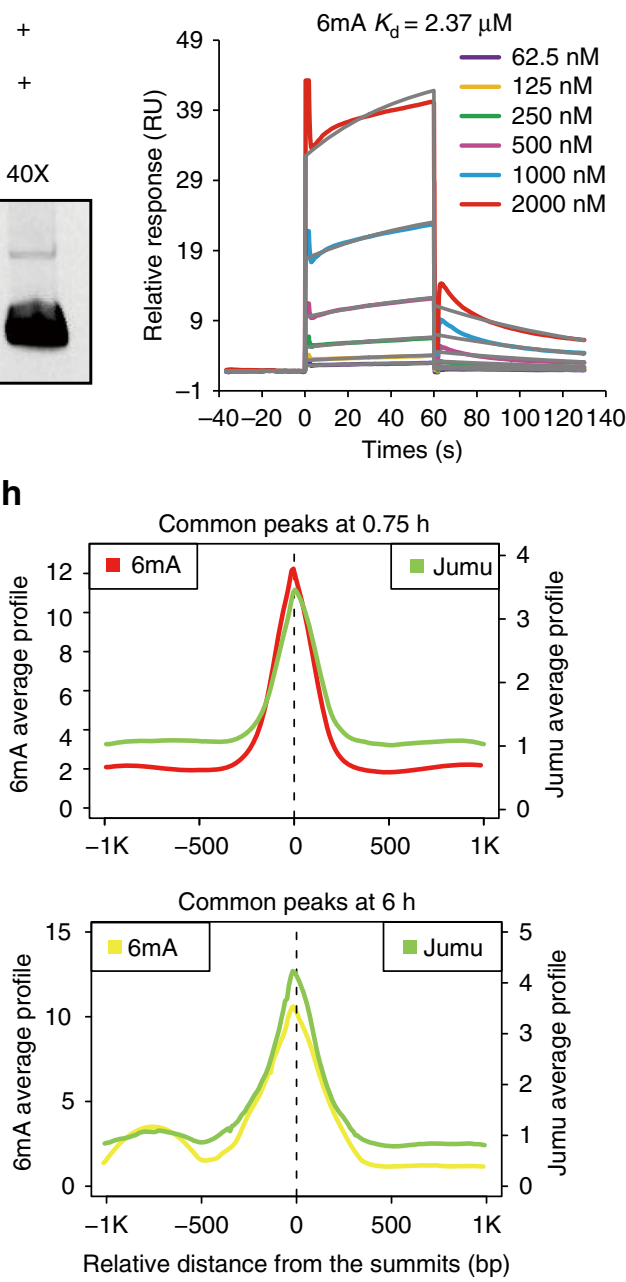

Relative distance from the summits (bp) 
Fig. 3 Jumu is a $6 \mathrm{~mA}$-marked DNA-binding protein. a Logo representations of Forkhead domain (FHD)-binding motifs identified in the $6 \mathrm{~mA}$ peaks at the 0.75 -h stage. For $6 \mathrm{~mA}$ peaks in 0.75 -h stage embryos, the number and percentage of peaks containing the motif and the E-value of motif occurrence were indicated. $\mathbf{b}$ Western blot assays using the anti-Jumu antibody against purified complexes. Complexes purified by the 6mA-containing DNA probes exhibited much higher Jumu signals than those purified by the control probes. c Electrophoretic Mobility Shift Assay (EMSA) showing the signals of the shifted probe bands in gradient dose of Jumu protein. The signals were progressively increased with gradient dose of Jumu protein present in the reactions. d Competitive EMSA in different titrations showing the different affinities of recombinant FHD of Jumu protein to dA-dsDNA and 6mA-dsDNA. e The competitive ratio of dA or $6 \mathrm{~mA}$ competitor in different titrations by the quantitative intensity of bands boxed (d). The one-tailed Student's $t$ test was used to analyze statistical variance. Data expressed as means of 4 independent experiments. Error bars indicate mean \pm s.d. ${ }^{\star \star \star} P<0.001$. $\mathbf{f}$ Surface plasmon resonance (SPR) measurements of the interaction between recombinant FHD of Jumu protein and dA/6mA-dsDNA. Equilibrium and kinetic constants were calculated by a global fit to 1:1 Langmuir binding model. RU, resonance units. g Overlap of $6 \mathrm{~mA}$ enrichment peaks and Jumu-binding peaks in 0.75-h embryos. $\mathbf{h}$ The average signal profile of $6 \mathrm{~mA}$ and Jumu-binding in common $6 \mathrm{~mA} / J u m u$ peaks in 0.75 - and 6-h stage embryos. Source data are provided as a Source Data file. n.s. not significant

and DMAD mutant brains (with high level of $6 \mathrm{~mA}$ ). Complexes immunoprecipitated by anti-Flag beads were then subjected to dot blot assays. As shown in Supplementary Figure 3c, Jumu captured much more of the $6 \mathrm{~mA}$ signal than the GFP control. We then performed high-throughput sequencing assays using genomic DNA from 0.75 - and 6-h embryos captured by the Flag-Jumu protein. By performing MACS2 analysis, we identified 3,079 and 921 Jumu-binding sites in the $0.75-$ and 6-h embryos, respectively. Strikingly, 68 and $78 \%$ of Jumu-binding peaks overlapped with the $6 \mathrm{~mA}$-enriched peak regions in the 0.75 - and 6-h embryos, respectively (Fig. 3g and Supplementary Figure 3d), and the summits of the Jumu-binding peaks almost overlapped with those of the $6 \mathrm{~mA}$ peaks (Fig. $3 \mathrm{~h}$ ). Importantly, the signal strength of $6 \mathrm{~mA}$ in the $6 \mathrm{~mA} / J u m u$ common peaks was much stronger than in the unique $6 \mathrm{~mA}$ peaks (Fig. $3 \mathrm{~h}$ and Supplementary Figure 3e). These results suggest that Jumu is a $6 \mathrm{~mA}-$ marked DNA-binding protein.

Jumu functions as a maternal factor in early embryos. Based on the above findings, we speculated that Jumu acts as a maternal factor to regulate early embryogenesis by binding $6 \mathrm{~mA}$ mark. We thus examined abundance of Jumu protein at various embryonic stages. As shown by western blot assays, the Jumu was present at relatively high levels in early embryos, but its levels were reduced at the 10-h stage (Fig. 4a). Of note, Jumu was also found at considerable levels in unfertilized eggs and ovaries (Fig. 4a), suggesting that Jumu were preloaded into mature oocyte. We then employed the CRISPR/Cas9 system to generate two jumu mutant alleles, jum $u^{1}$ and jumu $u^{2}$ (Fig. 4b). Genetic tests showed that about $45 \% \quad(n=116)$ of zygotic jumu mutants finally developed into adult flies, in which Jumu expression was completely abolished (Fig. 4c). Of note, although loss of zygotic Jumu reduced female fertility by affecting germline development, zygotic jumu mutant females still produced a few eggs, in which the maternal Jumu was absent (Fig. 4d). To determine the maternal role of Jumu, we used maternal jumu ${ }^{(\mathrm{M}-)}$ mutant eggs to perform further genetic experiments. As shown in hatching rate analysis, compared with the wild-type control, $\sim 72 \%$ of jum $u^{(\mathrm{M}-, \mathrm{Z}+)}$ embryos failed to hatch into larvae, when maternal jumu mutant eggs were fertilized by the wild-type sperm (Fig. $4 \mathrm{e}$ and Supplementary Figure 4a). Cuticle analysis showed that about $30 \%$ of dead mutant embryos (143/482) had displayed segmentation pattern with different numbers of segments, whereas about $70 \%$ of dead mutant embryos (339/482) even showed no segmentation phenotype (Fig. 4f). Further Immunostaining assays revealed that compared to the wild-type control, the hexagonalactin network was apparently disorganized in about 50\% (132/ 265) maternal jumu mutant embryos at the nuclear cycle 14 (Fig. 4g). Additionally, nuclear fallout phenotypes were observed in some jumu ${ }^{(\mathrm{M}-, \mathrm{Z}+)}$ mutant embryos (Fig. $4 \mathrm{~g}$ and Supplementary Figure $4 \mathrm{~b}$ ). These findings suggested a role of maternal Jumu in regulating cellularization in the early embryos. We noted that $\sim 86 \%$ of jumu ${ }^{(\mathrm{M}-, \mathrm{Z}-)}$ mutant displayed embryonic lethal phenotypes (Fig. 4e and Supplementary Figure 4a). Only a few of the jumu ${ }^{(\mathrm{M}-, \mathrm{Z}-)}$ mutant $(\sim 1.7 \%, 33 / 1934)$ reached adulthood. Notably, the jumu mutant sperm was sufficient to support normal embryogenesis when it was used to fertilize wild-type eggs (Fig. 4e and Supplementary Figure 4a). Because jumu( $u^{(M-, Z-)}$ embryos displayed severer embryonic lethal phenotypes than that in jumu $u^{(M-, Z+)}$ embryos, we examined the expression of Jumu in $j u m u^{(\mathrm{M}-, \mathrm{Z}+)}$ embryos at $0-1$ and $2-4 \mathrm{~h}$ stages, and found Jumu was zygotically expressed in embryos (Supplementary Figure 4c). These results suggest that Jumu plays important maternal roles during embryogenesis.

Maternal Jumu regulates early embryonic gene expression. Next, we tested whether the maternal Jumu controls the proper gene expression in early embryos, and generated RNA-seq data sets from $j u m u^{(\mathrm{M}-, \mathrm{Z}+)}, j u m u^{(\mathrm{M}-, \mathrm{Z}-)}$ and wild-type embryos at the 3-h stage. Because loss of maternal Jumu leads to embryonic lethal phenotypes, we first compared the gene expression profile of $j u m u^{(\mathrm{M}-, \mathrm{Z}+)}$ or $j u m u^{(\mathrm{M}-, \mathrm{Z}-)}$ mutant embryos with that of wild-type embryos, and identified 2198 genes that were significantly up/downregulated in both jumu $u^{(\mathrm{M}-, \mathrm{Z}+)}$ and jumu (M-,Z-) $^{(\mathrm{Z} \text { ) }}$ matant embryos (Fig. 5a). We defined these 2,198 genes as genes significantly regulated by the maternal Jumu (hereafter referred to as GSRJ), and divided the GSRJ into two groups, group 1 (up-regulated genes) and group 2 (downregulated genes). Gene ontology ( $\mathrm{GO}$ ) analysis revealed that some of GSRJ genes were development-related (Supplementary Figure 5a). Thus, lethality of maternal jumu mutant embryos could be, at least in part, attributed to abnormal expression of development-related genes. Of note, a number of the GSRJ genes encoding maternal transcription factors (e.g., Zelda ${ }^{20}$ and Lilli ${ }^{26}$ ) were found to be up-regulated in $3-\mathrm{h} j u m u^{(\mathrm{M}-, \mathrm{Z}-)}$ embryos. These findings suggest that loss of maternal Jumu changes gene expression pattern in early embryos.

Jumu regulates $6 \mathrm{~mA}$-marked zelda in early embryos. Given that Jumu binds to $6 \mathrm{~mA}$-marked DNA, we next investigated the role of Jumu in regulating its binding-target genes. We compared the Jumu-bound genes (definition seen in Methods) with the GSRJ, and found 189 Jumu-bound GSRJ (Fig. 5b). The remaining GSRJ without Jumu-binding sites were referred to as the Jumu-indirect GSRJ. By scanning the $6 \mathrm{~mA}$ peaks around the Jumu-bound GSRJ, we found that $95 \%(180 / 189)$ of Jumu-bound GSRJ were marked by $6 \mathrm{~mA}$, whereas only about $27 \%(534 / 2,009)$ of Jumu-indirect GSRJ were marked by $6 \mathrm{~mA}$ (Fig. $5 \mathrm{c}$ ).

To understand molecular mechanisms by which Jumu regulates early embryogenesis, we carefully examined the 189 Jumu-bound GSRJ, and found that some important genes, such as zelda, lilli, lola and Trf2, were marked by both $6 \mathrm{~mA}$ and 
a

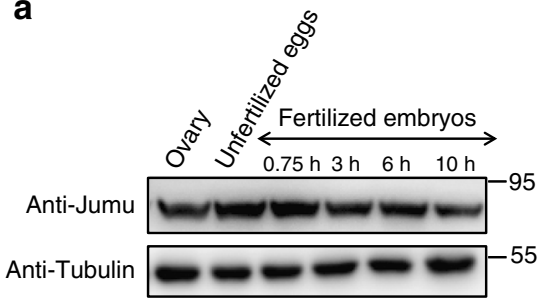

C

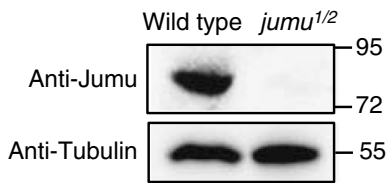

d

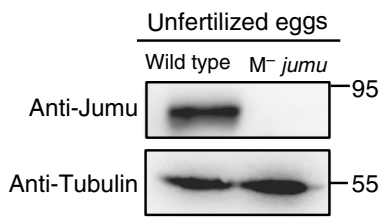

f

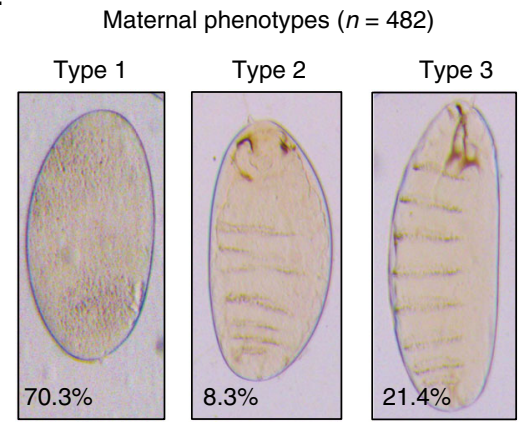

b

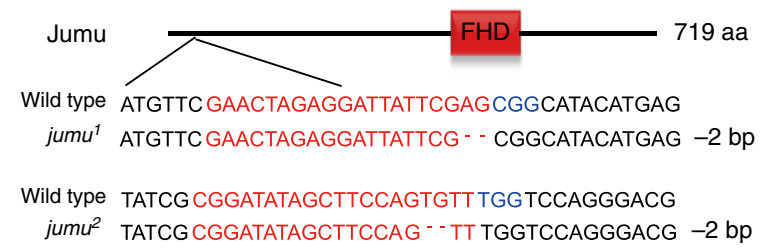

e

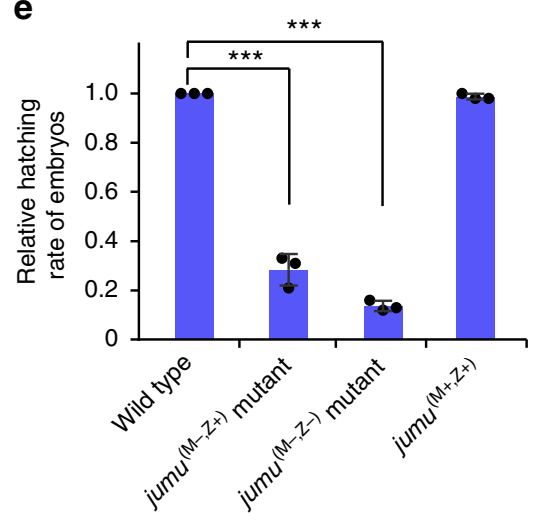

g

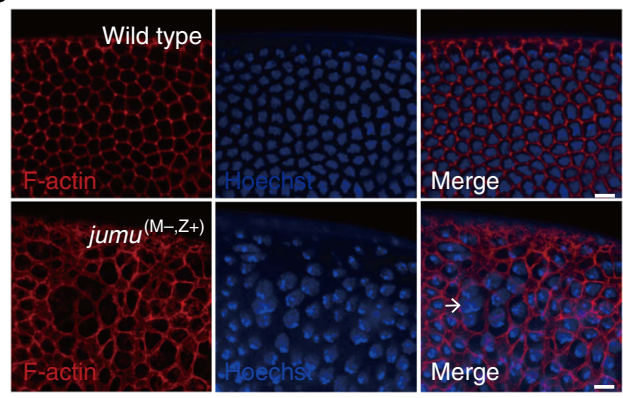

Fig. 4 Jumu functions as a maternal factor to regulate embryogenesis. a Western blot assays showing Jumu protein abundance in ovary, unfertilized eggs and embryos at various embryonic stages as indicated. b Two jumu mutant alleles, jumu ${ }^{1}$ and jumu ${ }^{2}$, were generated by the CRISPR/Cas9 system. c, d Western blot assays were performed to show expression levels of Jumu protein in wild type or jumu mutant ovaries (c), and in wild type or maternal jumu mutant eggs (d). e Relative hatching rate of embryos with indicated genotypes. The two-tailed Student's $t$ test was used to analyze statistical variance. Data expressed as means of 3 independent experiments. Error bars indicate mean \pm s.d. ${ }^{\star \star \star} P<0.001$. $\mathbf{f}$ Three typical phenotypes were observed in jumu maternal lethal embryos $\left(\mathrm{M}^{-}, \mathrm{Z}^{+}\right.$, jumu). $\mathbf{g}$ Wild type and maternal jumu mutant embryos were stained with phalloidin and Hoechst to visualize F-actin (red) and DNA (blue), respectively. Scale bar, $10 \mu \mathrm{m}$. Source data are provided as a Source Data file

Jumu-binding signals (Fig. 5d and Supplementary Figure 5b). Among these, we considered the zelda gene as being of particular interest because its product Zelda acts as a pioneer transcription factor essential for $\mathrm{MZT}^{20}$. In our RNA-seq data sets, zelda was expressed at considerable levels in 3-h wild-type embryos, but was further up-regulated in maternal Jumu mutant (Supplementary Figure $5 \mathrm{c}$ ). To validate the role of $6 \mathrm{~mA}$, we generated maternal DMAD mutant embryos (see Methods), and found that loss of the maternal DMAD caused embryonic lethal (Supplementary Figure 5d, e). Moreover, zelda was downregulated in maternal DMAD mutant (Supplementary Figure 5f, g). Thus, our findings suggest that Jumu negatively regulates zelda expression in early embryos likely through $6 \mathrm{~mA}$ modification.

We then examined embryos with overexpression of maternal Zelda. Consistent with previous findings ${ }^{22}$, Zelda overexpression caused partial embryonic lethal (710/1374), and some Zelda overexpression embryos displayed celluarization defects (Supplementary Figure 6a-e). Because zelda was up-regulated in maternal Jumu mutant embryos, we tested whether the embryonic lethal phenotype in the maternal Jumu mutant is attributed to abnormal expression of Zelda target genes. We generated the RNA-seq dataset of embryos (3-h stage) with overexpression of maternal Zelda. By integrating this dataset with the Zelda ChIP-seq data ${ }^{27}$, we identified 3,165 Zelda target genes. Notably, $78 \%(1715 / 2,198)$ of the GSRJ were Zelda-target genes, and $77 \%(146 / 189)$ of Jumu-bound GSRJ were Zeldatarget genes (Fig. 5e). Further analyses revealed that the group 1 genes of GSRJ up-regulated in maternal Jumu mutant were upregulated in Zelda overexpression samples, and likewise, the group 2 genes of GSRJ downregulated in maternal Jumu mutant were downregulated in Zelda overexpression samples (Fig. 5f, g). To obtain biological evidence, we generated zelda knockdown females carrying different transgene combinations, $\mathrm{P}$ $\{\text { nos-gvp }\}^{2 e d} / \mathrm{P}\{$ uas-zelda-RNAi $\}$ and $\mathrm{P}\{$ uasp-artmiR-zelda $\} / \mathrm{P}$ $\{\text { nos-gvp }\}^{3 e d}$, which produced embryos with partial and strong knockdown of maternal Zelda, respectively (Fig. 6a and Supplementary Figure 6f). Consistent with previous findings ${ }^{20}$, strong knockdown of maternal Zelda caused complete embryonic lethal (Supplementary Figure 6g), whereas partial 
a

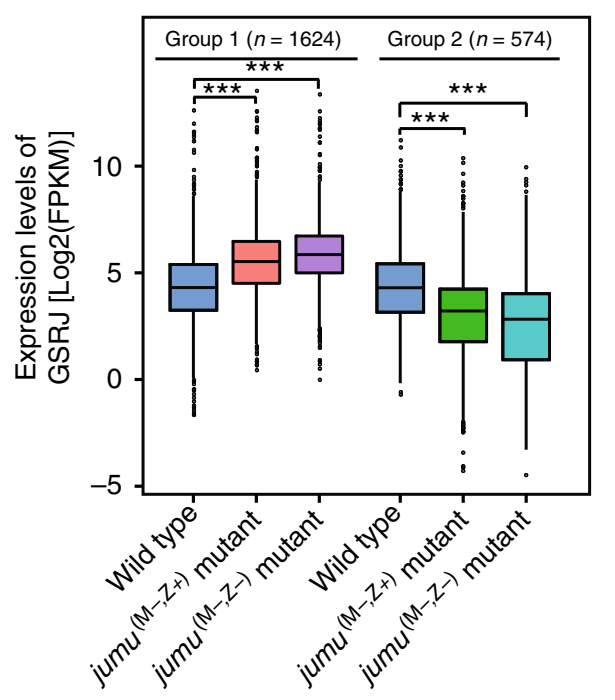

b

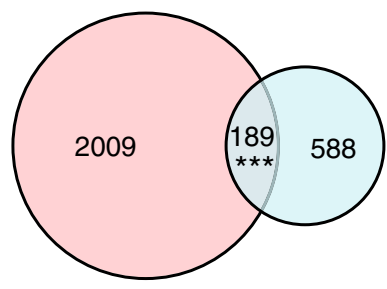

O GSRJ

Jumu-bound genes
C

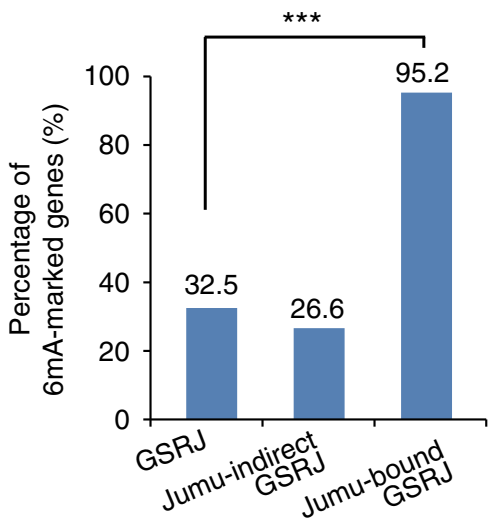

d

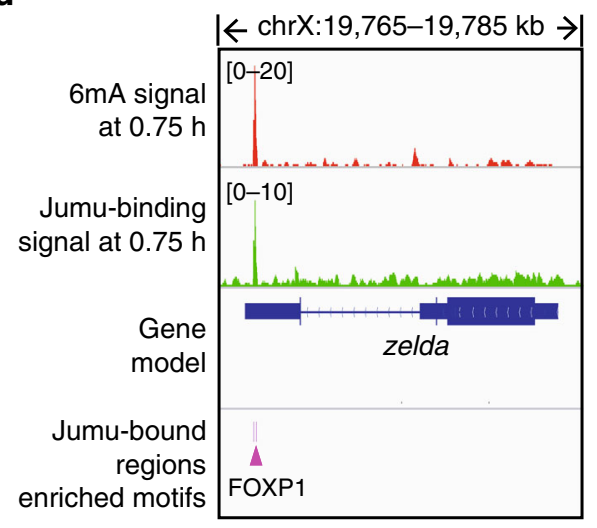

$\mathbf{f}$

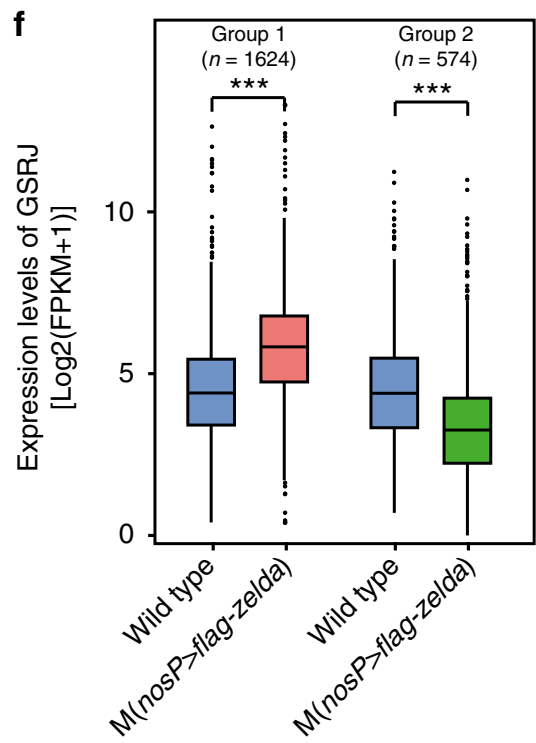

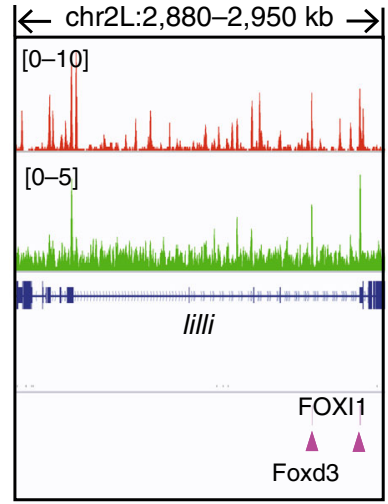

e

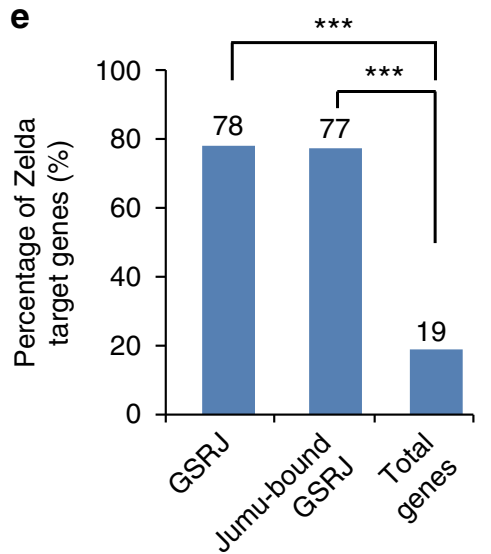

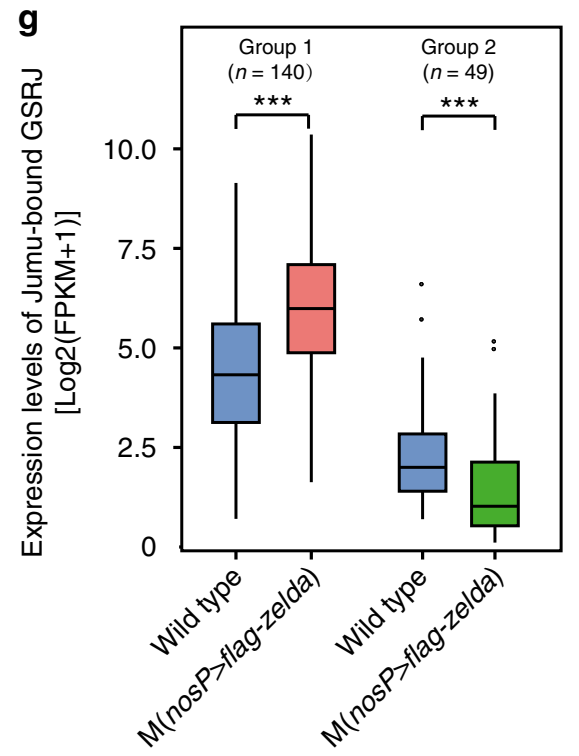

knockdown of maternal Zelda did not significantly affect embryonic development (Fig. 6b, c and Table 1). Notably, partial knockdown of maternal Zelda suppressed the embryonic lethal phenotype induced by loss of maternal jumu (Fig. 6b, c and Table 1). Thus, our findings suggest that Jumu regulates early embryogenesis, at least in part, through controlling $6 \mathrm{~mA}$ marked Zelda.

\section{Discussion}

The precise activation of zygotic genomes is essential for the normal embryogenesis; however, the molecular mechanisms that regulate this process have remained poorly understood. Our recent study showed that $6 \mathrm{~mA}$ dynamics in Drosophila early embryos almost corresponds to the process of MZT, raising a possibility that $6 \mathrm{~mA}$ forms an epigenetic mark contributing to 
Fig. 5 Jumu regulates embryonic gene expression via $6 \mathrm{~mA}$ modification. a A total of 1624 genes of GSRJ (group 1) were significantly up-regulated and 574 genes of GSRJ (group 2) were significantly downregulated in jumu mutant embryos. $P$ values were calculated by one-tailed Student's $t$ test. ${ }^{\star \star \star} P<0.001$. GSRJ, genes significantly regulated by the maternal Jumu. b Overlap of Jumu-bound genes and GSRJ. $P$ value $(P=7.3 e-06)$ was calculated by one-tailed hypergeometric test. ${ }^{\star \star \star} P<0.001$. c Percentage of $6 \mathrm{~mA}$-marked genes in different gene sets as indicated. $P$ value was calculated by one-tailed hypergeometric test. ${ }^{\star \star \star} P<0.001$. d Examples for $6 \mathrm{~mA}$ and Jumu-binding signals around important gene (zelda, lilli). The enriched motifs in Jumu-bound regions were shown in figures. e Percentage of Zelda target genes in different gene sets as indicated. $P$ value was calculated by one-tailed hypergeometric test. ${ }^{\star \star \star} P<0.001$. $\mathbf{A}$ A total of 1624 genes of GSRJ (group 1) were upregulated and 574 genes of GSRJ (group 2) were downregulated in zelda overexpressed embryos. $P$ values were calculated by one-tailed Student's $t$ test. ${ }^{\star \star \star} P<0.001$. $\mathbf{g}$ Jumu-bound group 1 GSRJ were upregulated and Jumu-bound group 2 GSRJ were downregulated in zelda overexpressed embryos. $P$ values were calculated by one-tailed Student's $t$ test. ${ }^{* \star *} P<0.001$. For all boxplots, the centre line indicates the median, the bottom and top of the box show the first and third quartiles of the data, and the whiskers show the minimum and maximum values. Source data are provided as a Source Data file

a

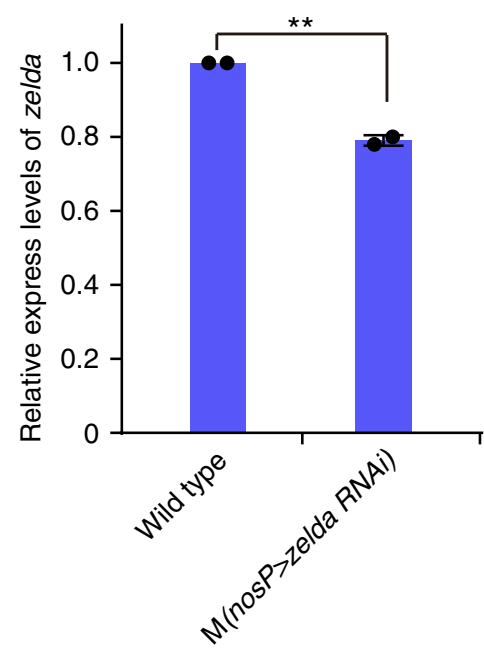

b

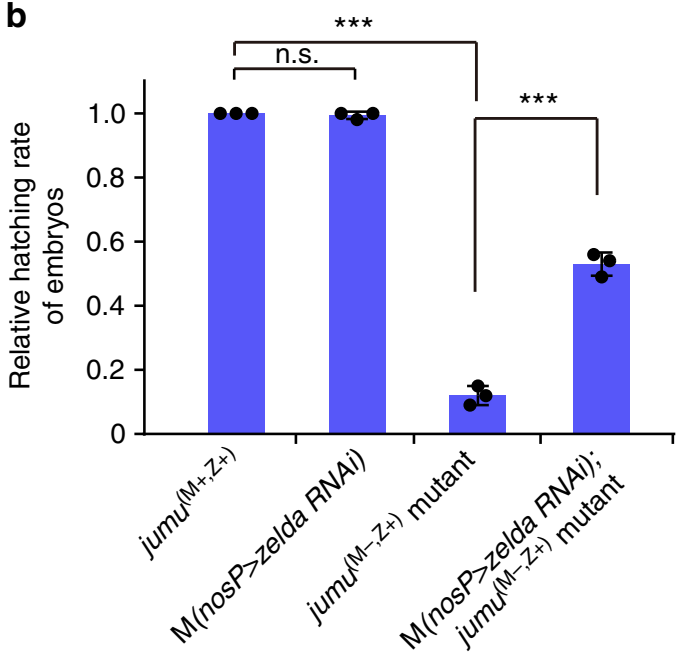

C

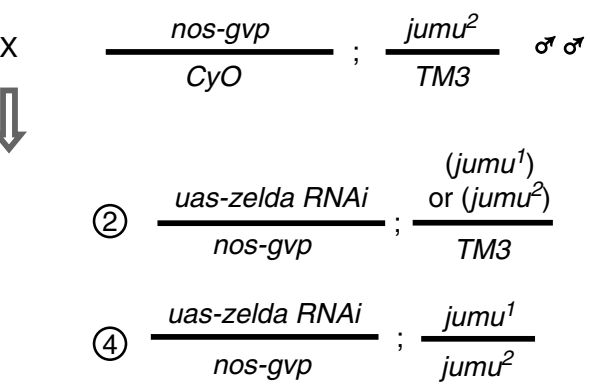

X $w^{1118}$ or or

$\downarrow$
(2) $\mathrm{M}($ nosP $>$ zelda $R N A i)$
(4) $\mathrm{M}($ nosP $>$ zelda $R N A i) ; j u m u^{(\mathrm{M}-, \mathrm{Z}+)}$
F2:
(1) $j u m u^{(\mathrm{M}+, \mathrm{Z}+)}$
(3) $j u m u^{\left(\mathrm{M}-, \mathrm{Z}_{+}\right)}$
Statistics of hatching rate of embryos

Fig. 6 Maternal Zelda overexpression causes partial embryonic lethal. a Quantitative RT-PCR experiments were used to analyze the effectiveness of zelda knockdown in 0.75-h stage embryos. The two-tailed Student's $t$ test was used to analyze statistical variance. Data expressed as means of 2 independent experiments. Error bars indicate mean \pm s.d. ${ }^{\star \star} P<0.01$. b Relative hatching rate of embryos with indicated genotypes. The two-tailed Student's $t$ test was used to analyze statistical variance. Data expressed as means of 3 independent experiments. Error bars indicate mean $\pm \mathrm{s}$.d. ${ }^{\star \star \star} P<0.001$. c Females with indicated genotype (uas-zelda RNAi/CyO; jumu ${ }^{1} / T M 3$ ) were crossed with males (nos-gvp/CyO;jumu²/TM3), the eggs produced by female progenies were then fertilized by the wild-type sperm. Unhatched embryos and larvae with indicated genotypes were counted separately. Source data are provided as a Source Data file. n.s. not significant 
Table 1 Hatching rate of embryos with indicated genotypes

Genotype of embryos

jumu $(\mathrm{M}+, \mathrm{Z}+)$

$\mathrm{M}($ nos $P>$ zelda $R N A i)$

jumu $(\mathrm{M}-, \mathrm{Z}+)$ mutant

$\mathrm{M}($ nosP $>$ zelda $\left.R N A i) ; j u m u^{(M-,} \mathrm{Z}_{+}\right)$mutant
Hatching rate

Replicate 1

$0.91\left(480 / 530^{\star}\right)$

$0.91\left(1018 / 1114^{\star}\right)$

$0.11\left(32 / 296^{*}\right)$

$0.51\left(338 / 668^{\star}\right)$
Replicate 2

$0.90\left(285 / 317^{\star}\right)$

$0.90\left(130 / 145^{\star}\right)$

$0.14\left(11 / 81^{\star}\right)$

$0.49\left(512 / 1047^{\star}\right)$
Replicate 3

$0.86\left(425 / 494^{\star}\right)$

$0.84\left(323 / 383^{\star}\right)$

$0.08\left(27 / 343^{\star}\right)$

$0.42\left(286 / 681^{\star}\right)$

*Sample size

MZT. In this study, we shed light on this intriguing issue, and found $6 \mathrm{~mA}$ can mark and associates with zygotic gene expression in early embryos. Importantly, we found that the Fox family protein Jumu bound $6 \mathrm{~mA}$-marked gene and functioned as a maternal factor to control the proper activation of zygotic genomes. Thus, our findings support a notion that $6 \mathrm{~mA}$-formed epigenetic code can be read by the maternal factor, thus controlling normal embryogenesis.

It has been suggested that Zelda and Zelda-like proteins function as pioneer transcription factors to initiate zygotic genome activation $^{20}$. Interestingly, we found that zelda was marked with $6 \mathrm{~mA}$ and regulated by Jumu. Bioinformatics analysis showed that $78 \%(1715 / 2,198)$ of the GSRJ were also Zelda's targets. Consistently, genetic experiments showed that maternal overexpression of Zelda caused embryonic lethal phenotype, which mimics that observed in Jumu maternal mutant embryos. Importantly, we found that partial knockdown of Zelda significantly suppressed the embryonic lethal phenotype induced by loss of maternal Jumu. These findings strongly suggested that Zelda is one of critical target genes of Jumu, and that Jumu regulates the proper zygotic genome activation, at least in part, through regulating Zelda (Fig. 7a). Previous studies have suggested that loss of maternal Zelda leads to either down-regulation or upregulation of target genes in Drosophila embryos, suggesting that Zelda regulates gene expression in either direct or indirect manner, although Zelda is a positive regulator of gene expression. For the case of indirect targets, Zelda could activate a set of miRNAs, which inhibit sets of downstream target genes ${ }^{20,28}$. In addition to zelda, our results suggest that other important genes, such as lilli and lola, were marked with $6 \mathrm{~mA}$ and regulated by Jumu. Based on our data analysis, we propose a model by which the maternal factor Jumu specifically reads a $6 \mathrm{~mA}$-based epigenetic code to control other transcription factors including Zelda, thereby ensuring the proper embryogenesis (Fig. 7b). Of note, Jumu is required for both germline development and embryogenesis; however, the actions of Jumu appear to be different between two biological contexts (Supplementary Figure $7 \mathrm{a}-\mathrm{g}$ ). In the context of early embryos, loss of maternal Jumu led to upregulation of Zelda, and loss of Jumu and overexpression of maternal Zelda caused similar embryonic lethal phenotypes. Importantly, transcriptomic profiles of GSRJ in maternal Jumu mutant were very similar to that in maternal Zelda overexpression samples. By contrast, in context of germline, knockdown or overexpression of Zelda in germ cells had no apparent effects on the normal oogenesis, suggesting that Zelda is dispensable for germline development (Supplementary Figure $7 \mathrm{~h}$ ).

Here we identified a Fox family protein Jumu functions as a $6 \mathrm{~mA}$ reader to regulate gene expression. However, it still remains unknown about whether $6 \mathrm{~mA}$ modification has a role to inhibit the binding of protein-DNA. Nevertheless, because Fox family proteins have conserved roles in controlling embryonic development and tissue homeostasis, misregulation of some Fox family proteins has been implicated in many human diseases including cancers $^{29,30}$. It will be interesting to investigate how Fox family proteins function in concert with $6 \mathrm{~mA}$ epigenetic mark to regulate development in mammals and in human diseases.

\section{Methods}

Drosophila strains. Fly stocks were maintained under standard culture conditions. The Drosophila $w^{1118}$ strain was used for collecting the wild-type embryos in this study. DMAD null flies, $D M A D^{1}$ and $D M A D^{2}$, were described previously ${ }^{11}$. The following strains were generated in this study: (1) Two jumu mutant alleles, jumu and $j u m u^{2}$, were generated by the following method ${ }^{11}$. Briefly, the target DNA sequence selected for CRISPR RNA-guided Cas9 nuclease was predicted by crisper/ cas target software (http://zifit.partners.org/ZiFiT). The templates for gRNA in vitro transcription were amplified from pMD19T-gRNA scaffold. Then the gRNA was in vitro transcribed through run-off reactions and injected into vasaCas 9 embryos. The flies were incubated under standard culture conditions and outcrossed for further screening by T7E1 enzyme digestion and sequencing; (2) The transgene line, $\mathrm{P}\{$ uasp-flag-zelda $\}$, in which the full-length zelda was inserted into UASp-flag vectors; (3) The zelda knockdown line, $\mathrm{P}\{$ uasp-artmiR-zelda $\}$ was generated by the following method. A pair of designed primers was denatured and annealed to form short dsDNA, which were then digested by NheI and EcoRI enzymes to insert into UASp BX vectors. The $w^{1118}$ strain was used as the host for $\mathrm{P}$ element-mediated transformations. $\mathrm{P}\{$ uas-zelda $R N A i\}$ was obtained from Tsinghua fly center. $\mathrm{P}\{\text { nos-gvp }\}^{2 e d}$ and $\mathrm{P}\{\text { nos-gvp }\}^{3 e d}$ were used as a maternal driver. The detailed information of primers was described in the Supplementary Table 1.

Embryo preparation. The flies were maintained in large population cages in an incubator at standard conditions $\left(25^{\circ} \mathrm{C}\right)$. As the protocol for collecting embryos, the well-fed flies were used to lay eggs in bottles, each of which was covered by a petri dish with agar gel. In this work, six students and postdocs carefully collected and examined embryos under the light microscopes. For the 0.75 -h stage, embryos were collected for $30 \mathrm{~min}$, and then allowed to develop for $15 \mathrm{~min}$. Older embryos were removed using the light microscopes ${ }^{27}$ and the embryos were cleaned using washing buffer ( $1 \mathrm{x}$ PBS) to avoid contamination. All actions were stopped at the $0.75-\mathrm{h}$, at which the samples were immediately put into liquid nitrogen and stored at $-80^{\circ} \mathrm{C}$. Likewise, the ranges of $3-\mathrm{h}$ and $6-\mathrm{h}$ are $2.5-3 \mathrm{~h}$ and $5.5-6 \mathrm{~h}$, respectively.

Purification of genomic DNA. Drosophila samples were collected and dissected with indicated stages and tissues. Genomic DNA was extracted using a Wizard genomic DNA purification kit (Promega) following the manufacturer's instructions.

6mA-DNA-IP-Seq. Purified genomic DNA was sonicated to 200 base pairs (bp). 5 $\mu \mathrm{g}$ of fragmented genomic DNA was immunoprecipitated with $5 \mu \mathrm{g}$ of rabbit $6 \mathrm{~mA}$ antibody (Abcam) or rabbit IgG overnight at $4{ }^{\circ} \mathrm{C}$ in a final volume of $500 \mu \mathrm{l}$ immunoprecipitation buffer (10 mM sodium phosphate, $\mathrm{pH} 7.0,140 \mathrm{mM} \mathrm{NaCl}$, $0.05 \%$ Triton $\mathrm{X}-100$ ). The mixture was incubated with $50 \mu \mathrm{l}$ protein $\mathrm{A} / \mathrm{G}$ agarose beads for $2 \mathrm{~h}$ at $4{ }^{\circ} \mathrm{C}$ and then washed three times with $1 \mathrm{ml}$ of immunoprecipitation buffer. The beads were then treated with proteinase $\mathrm{K}$ and the methylated DNA was purified by phenol-chloroform extraction followed by ethanol precipitation for library construction. End repair, 3'-adenylation, adaptor ligation, and PCR amplification were performed according to the Illumina TruSeq DNA sample preparation procedures. The libraries were sequenced using an Illumina HiSeq $2500 \mathrm{~V} 4$ platform. Of note, the anti-6mA used in this study can efficiently capture either single-strand or double-strand genomic DNA (Supplementary Figure 1b, c)

It is worth to note that both IgG- and input-based controls have been widely used to call peaks in DNA-IP-seq experiments in many studies ${ }^{31,32}$. In this study, we found that there was no significant bias between two approaches. For example, at the 0.75 -h stage, we found that $96 \%$ of Input-controlled $6 \mathrm{~mA}$ peaks were overlapped with IgG-controlled $6 \mathrm{~mA}$ peaks (Supplementary Figure 1f).

RNA sequencing (RNA-seq). Total RNAs were isolated from embryos at the indicated stages using Trizol reagent (Thermo Fisher). The mRNAs were purified using a Dynabeads mRNA DIRECT Kit (Thermo Fisher) according to the user 
a

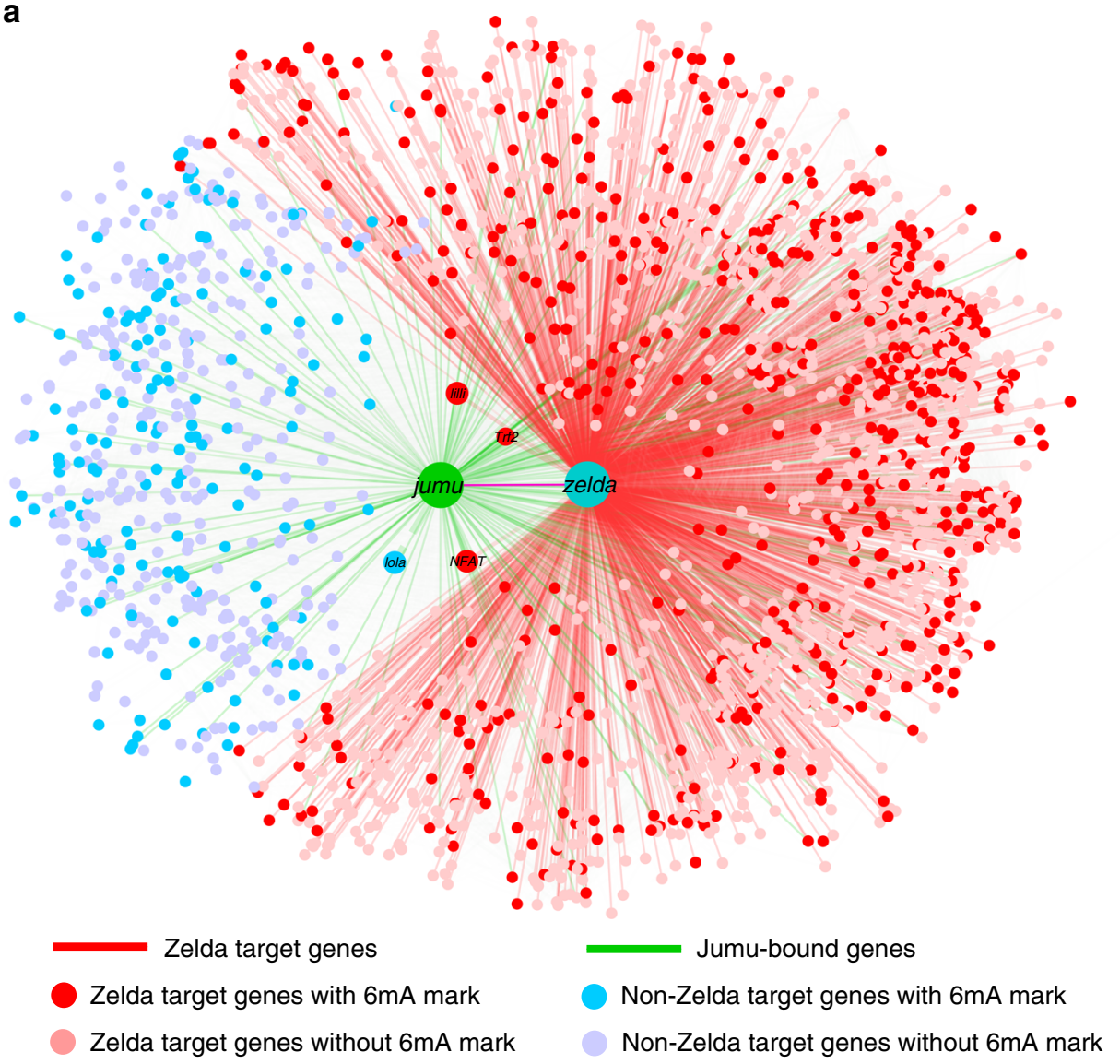

b

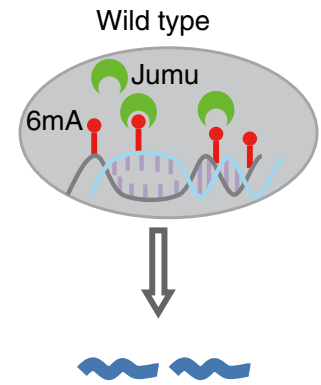

Normal expression of zelda and other factors
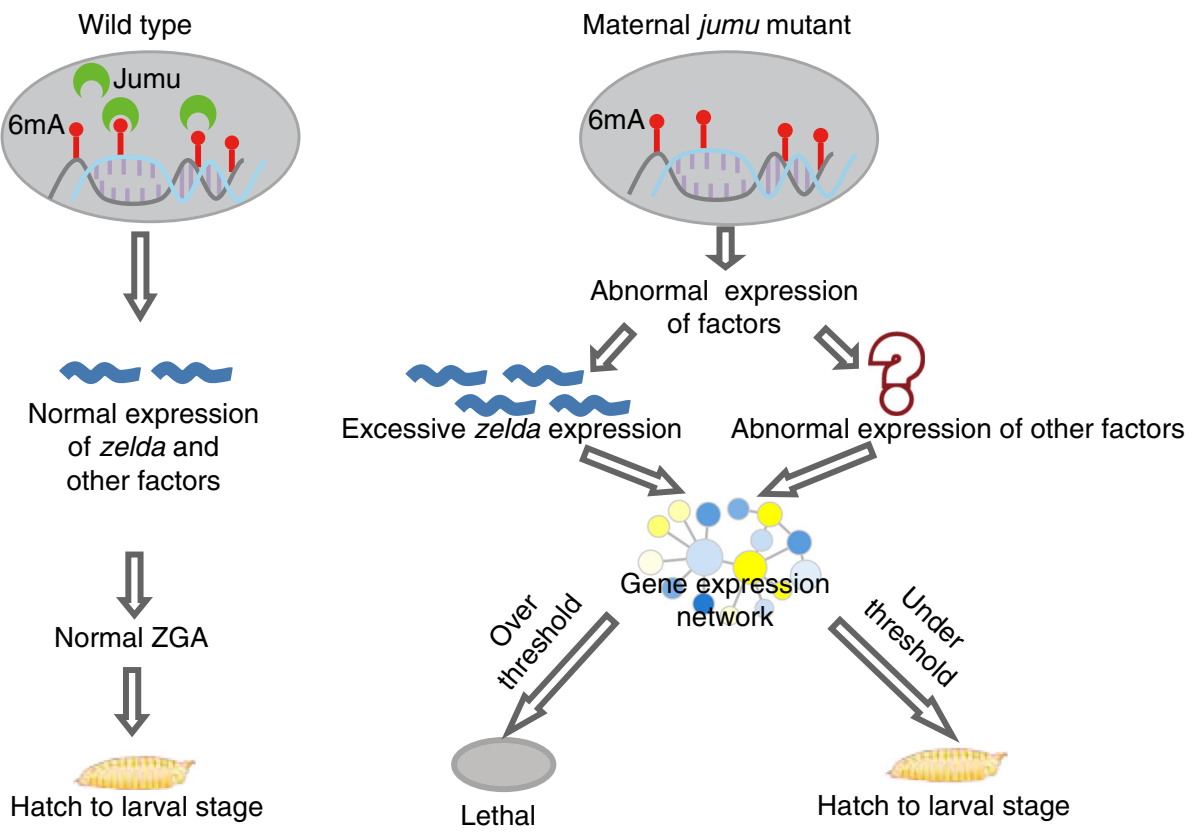

Fig. 7 Regulatory gene network of GSRJ mediated by Jumu. a The regulatory network by which Jumu regulates embryonic gene expression in Zeldadependent and Zelda-independent manners. The Jumu/Zelda target genes were as defined in the main text. b Model for Jumu as a $6 \mathrm{~mA}$ reader that regulates embryonic gene expression. ZGA, zygotic genome activation. Source data are provided as a Source Data file

manual. Illumina sequencing libraries were constructed according to the manufacturer's instructions and sequenced using an Illumina HiSeq 2500 V4 or NovaSeq platform.

Protein-DNA interaction and high-throughput sequencing. Plasmids were transfected into HEK293T cells using polyethyleneimine (PEI, Sigma). The cells were treated with lysis buffer $(50 \mathrm{mM}$ Tris- $\mathrm{HCl}, \mathrm{pH} 7.4,500 \mathrm{mM} \mathrm{NaCl}, 1 \% \mathrm{NP}-40$ protease inhibitor) after culturing for $48 \mathrm{~h}$. Following centrifugation $(20,000 \times g)$ for $10 \mathrm{~min}$ at $4{ }^{\circ} \mathrm{C}$, the supernatants were mixed with anti-FLAG M2 affinity beads (Sigma) and incubated for 3 -h at $4{ }^{\circ} \mathrm{C}$. Then the beads were washed twice with lysis buffer and twice with TBS (20 mM Tris- $\mathrm{HCl}, \mathrm{pH} 7.4,150 \mathrm{mM} \mathrm{NaCl})$. Five micrograms of fragmented genomic DNA and immunoprecipitation buffer to a 
final volume of $500 \mu \mathrm{l}$ was added to the beads and held overnight at $4{ }^{\circ} \mathrm{C}$. Then the mixture was washed three times with $1 \mathrm{ml}$ immunoprecipitation buffer and the beads were treated with proteinase $\mathrm{K}$. The bound DNA was purified by phenol-chloroform extraction followed by ethanol precipitation. Sequencing libraries were prepared according to the Illumina TruSeq DNA sample preparation procedures and high-throughput sequencing was performed using an Illumina HiSeq 2500 V4 platform.

Synthesis of DNA probes. Unlabeled or biotin/FAM-labeled dA/6mA dsDNA probes were synthesized using PCR reactions with 2'-deoxyadenosine-5'-triphosphate (dATP) or N6-methyl-2'-deoxyadenosine- $5^{\prime}$-triphosphate ( $\left.\mathrm{dm}^{6} \mathrm{ATP}\right)$ (Trilink) in place of dATP. The PCR products were purified using Universal DNA Purification Kit (Tiangen). The sequences of the dsDNA substrates are listed below:

\section{Probe. 5'-GCTATTTATGCGTTCTTTTCTTCTTTCTCATTTCTATATTTA- TAGTGTGTGTGTGTGTGTGGGTGTGTTTGTTGTATGTGGTTAAA-} TATGCTTCGTTTTTGTTTAATTGGTTTTTGTTTTTGTGATGATCATGGG-3

DNA pull-downs and Mass Spectrometry. Embryos were homogenized in STMDPS- $250 \mathrm{mM}$ buffer $(0.25 \mathrm{M}$ sucrose, $25 \mathrm{mM} \mathrm{KCl}, 5 \mathrm{mM} \mathrm{MgCl} 2,50 \mathrm{mM}$ Tris$\mathrm{HCl}, \mathrm{pH} 7.4,1 \mathrm{mM}$ DTT). After $75-\mu \mathrm{m}$ filtration, the crude nuclear pellet was obtained by centrifugation at $800 \times g$ for $10 \mathrm{~min}$. The pellet was then resuspended in STMDPS-1.8 M buffer (1.8 M sucrose) layered over STMDPS-2M buffer $(2 \mathrm{M}$ sucrose) and centrifuged at $100,000 \times g$ for $1 \mathrm{~h}$. The nuclei were lysed in nuclear lysis buffer ( $420 \mathrm{mM} \mathrm{NaCl}, 20 \mathrm{mM}$ HEPES, pH 7.9, $20 \% \mathrm{v} / \mathrm{v}$ glycerol, $2 \mathrm{mM} \mathrm{MgCl}_{2}$, $0.2 \mathrm{mM}$ EDTA, $0.1 \% \mathrm{NP} 40$, complete protease inhibitor without EDTA (Roche) and $0.5 \mathrm{mM} \mathrm{DTT}$ ) and centrifuged at $100,000 \times g$ for $1 \mathrm{~h}$. For each DNA pulldown, $10 \mu \mathrm{g}$ of biotin-labeled dA/6mA dsDNA probes was immobilized on $60 \mu \mathrm{l}$ of streptavidin beads (GE) by incubating for $1 \mathrm{~h}$ at room temperature in DNAbinding buffer ( $10 \mathrm{mM}$ sodium phosphate, $\mathrm{pH} 7.0,140 \mathrm{mM} \mathrm{NaCl}, 0.05 \% \mathrm{NP} 40)$. The beads were then incubated with $400 \mu \mathrm{g}$ of nuclear extract in a total volume of $600 \mu \mathrm{l}$ of protein binding buffer $(50 \mathrm{mM}$ Tris- $\mathrm{HCl}, \mathrm{pH} 8,150 \mathrm{mM} \mathrm{NaCl}, 1 \mathrm{mM}$ DTT, $0.25 \%$ NP40 and complete protease inhibitors) for $2 \mathrm{~h}$. After washing three times with protein binding buffer, proteins were eluted and digested using Trypsin (Promega), and then subjected to LC-MS/MS assays. The resulting MS/MS data were processed using Thermo Proteome discovery (version 1.4.1.14), and the tandem mass spectra were searched against the UniProt Drosophila proteome database ${ }^{33}$.

Electrophoretic mobility shift assay (EMSA). EMSAs were performed using both $5^{\prime}$ and $3^{\prime}$-FAM-labeled dsDNA probes and GST-Jumu FKH domain protein (amino acid residue 391-540). Briefly, the GST-Jumu FKH protein was expressed in Escherichia coli strain BL21 (DE3) and purified by GST affinity purification. The FAM-dA/6mA dsDNA probes $(15 \mathrm{nM})$ were incubated with a gradient dose of 1,2 and $4 \mu \mathrm{M}$ GST-Jumu FKH protein in buffer containing $20 \mathrm{mM}$ HEPES, pH 7.5, $50 \mathrm{mM} \mathrm{KCl}, 1 \mathrm{mM}$ DDT, $1 \mathrm{mM}$ EDTA, $0.01 \mathrm{mg} / \mathrm{ml} \mathrm{BSA}$, 2\% ficoll 400 and $5 \mathrm{ng} / \mu \mathrm{l}$ Poly (dI:dC) at $25^{\circ} \mathrm{C}$ for $20 \mathrm{~min}$. For competitive EMSAs, $2 \mu \mathrm{M}$ GST-Jumu FKH protein and indicated molar excess of the competitive dA/6mA dsDNA (10, 20 and 40 -fold) were used. Reaction products were subjected to $6 \%$ native polyacrylamide gel and run forlh at $80 \mathrm{~V}$ in $0.5 \times \mathrm{TBE}$ buffer. The gels were scanned by Typhoon FLA 9500 (GE Healthcare). The quantitation of band intensity in 3e was measured using ImageJ software.

Surface plasmon resonance (SPR) measurements. Biotinylated-dA/6mA DNA probes were captured on SA sensor chip (91-97 response units). Another blank flow cell was used as reference to correct for instrumental and concentration effect. SPR experiments were performed using Biacore $8 \mathrm{~K}$ instrument (GE Healthcare) in running buffer containing $20 \mathrm{mM}$ Tris-Cl, $\mathrm{pH} 8.0,150 \mathrm{mM} \mathrm{NaCl}$ and $0.005 \%$ Tween 20 at $25^{\circ} \mathrm{C}$. Jumu FKH proteins with increasing concentrations were injected into the $\mathrm{dA} / 6 \mathrm{~mA}$ probes surface and blank flow cell for $1 \mathrm{~min}$ at a flow rate of $30 \mu \mathrm{l} / \mathrm{min}$, dissociated for $1 \mathrm{~min}$ in running buffer. Equilibrium and kinetic constants were calculated by a global fit to 1:1 Langmuir binding model (Biacore 8 $\mathrm{K}$ evaluation software).

Western blot analysis. Western blots were performed by using standard protocols. The following reagents were used: mouse anti- $\beta$-Tubulin (1:2,000; Cwbio); anti-Flag M2 antibody (1:5,000; Sigma); mouse anti-Jumu (1:1000). The antibody against Jumu was generated by immunizing mice with a recombinant protein GSTJumu (amino acids 460-700) that was produced in E. coli. Uncropped blots are provided in Source Data file.

Dot blot assay. Different DNA samples were denatured at $95^{\circ} \mathrm{C}$ for $10 \mathrm{~min}$ and spotted on nitrocellulose membranes. DNA was cross-linked to the membranes by UV irradiation and the membranes were blocked in 5\% BSA in PBS containing $0.5 \%$ Tween 20 (PBST) for $1 \mathrm{~h}$ at room temperature. The membranes were then incubated with a $1: 10,000$ dilution of a $6 \mathrm{~mA}$ antibody (Abcam) overnight at $4{ }^{\circ} \mathrm{C}$. After three rounds of washes with PBST, the membranes were incubated with a 1:5,000 dilution of HRP-conjugated anti-rabbit IgG secondary antibody. The membranes were then washed with PBST three times and treated with enhanced chemiluminescence. Uncropped blots are provided in Source Data file.

Analysis of hatching rate and cuticle preparation of embryos. Embryos were collected for $24 \mathrm{~h}$ at $25^{\circ} \mathrm{C}$, then removed from the adults and allowed to develop for another $36 \mathrm{~h}$. Then, unhatched embryos and larvae were counted separately. For cuticle preparation, unhatched embryos were washed and dechorionated with $50 \%$ bleach. Dechorionated embryos were mounted on slides with Hoyer's medium, and baked at $60^{\circ} \mathrm{C}$ overnight. Embryos were viewed and scored using a Leica MZ16 stereo zoom microscope.

Quantitative Real-Time PCR Analysis. Embryos were collected at the indicated stages for RNA extraction. Total RNA was extracted using Trizol reagent (Thermo Fisher) and cDNA was generated with a FastQuant RT Kit (with gDNase) (Tiangen). Real-time quantitative PCR reactions were performed in triplicate on a LightCycler_480 Real-time PCR instrument (Roche) using the UltraSYBR mixture (Cwbio). Actin5C was used as the constitutive control for normalization of candidate gene expression. Relative gene expression was calculated using the $\Delta \Delta \mathrm{Ct}$ method following the manufacturer's instructions. The qRT-PCR primers for RNA quantification are listed in the Supplementary Table 1.

Embryo staining. Embryos were fixed in fixation buffer (4\% formaldehyde and $0.3 \%$ Tween-20 in PBS) for 30 min and washed in PBST (0.3\% Tween-20 in PBS) for 15 min. F-actin and DNA were visualized by Alexa Fluor ${ }^{\text {mot }} 546$ Phalloidin (1:30, Thermo Fisher) and by Hoechst (1:2,000, Sigma), respectively. They were added to PBST and incubated with embryos overnight at $4{ }^{\circ} \mathrm{C}$, followed by washing for three times (10 min per time) in PBST. The images were collected on a Zeiss LSM 710 Meta confocal microscopy.

Gene knockdown in Drosophila embryos. dsRNA fragments corresponding to $D M A D$ and $g f p$ mRNAs were synthesized in a PCR reaction and then fused to the T7 RNA polymerase binding site at both $5^{\prime}$ and $3^{\prime}$ ends. These were then used to generate dsRNA in vitro using the RiboMAX ${ }^{\text {st }}$ Large Scale RNA kit (Promega) following the manufacturer's instructions. The dsRNA $(1 \mu \mathrm{g} / \mu \mathrm{l})$ was injected into $w^{1118}$ embryos. The embryos were incubated at room temperature for turnover of the target protein. The primers used for the generation of DMAD and $g f p$ DNA fragments are shown in the Supplementary Table 1.

6mA-DNA-IP-Seq data analysis for Drosophila. After performing DNA immunoprecipitation (DNA-IP) experiments using an anti-6mA antibody, $125 \mathrm{bp}$ paired-end high-throughput sequencing was performed on an Illumina HiSeq 2500 V4 platform by Berry Genomics company. In total, 15.6, 11.7, 11.1, 6.8, 9.2 and 15.7 million paired-end reads were obtained from $0.75-\mathrm{h} 6 \mathrm{~mA}$ IP and IgG samples, 3-h 6mA IP and IgG samples, 6-h 6mA IP and IgG samples from Drosophila wildtype embryos, respectively. Adaptor sequences were trimmed, and the reads were then mapped to the Drosophila reference genome (UCSC version dm6, BDGP Release 6, unlocalized scaffolds excluded). Bowtie2 software ${ }^{34}$ was used with parameters "--no-mixed --no-discordant --non-deterministic --very-sensitivelocal" to align the paired-end reads. The callpeak module in MACS2 ${ }^{35}$ was used to identify enrichment peaks with parameters "-f BAMPE - B" and a default q value (FDR) cutoff of 0.05 , with IgG as a control. In total, 17,528, 4,363, 2,447 6mA peaks were identified at the $0.75-, 3-, 6$-h wild-type embryos, respectively. To compare $6 \mathrm{~mA}$ signals from different samples, a fold enrichment method was used to normalize the signals, and the MACS2 bdgcmp module was used to generate the fold enrichment score track with parameters "-m FE - $\mathrm{p} 2$ " by comparing wild type and IgG signal tracks in bedGraph.

Protein-DNA interaction and high-throughput sequencing. Analysis of the high-throughput sequencing data for Jumu protein-DNA interaction was performed according to the method used to analyze the 6mA-DNA-IP-Seq data.

Demonstration of the signal of $6 \mathrm{~mA}$ and Jumu-binding. Demonstration of the average signal profile of $6 \mathrm{~mA}$ and Jumu-binding was performed using the SitePro tool from the Cis-regulatory Element Annotation System (CEAS) ${ }^{36}$. The signal distribution on the genome was visualized using the Integrative Genomics Viewer (IGV) software ${ }^{37}$

Peak annotation. The genomic distribution of $6 \mathrm{~mA}$ peaks was annotated by HOMER $^{38}$. We noted that while about $80 \%$ of $6 \mathrm{~mA}$ peaks were less than $400 \mathrm{bp}$ in length, $3.2 \%$ of 0.75 -h peaks and $1.4 \%$ of 3 -h peaks had a width longer than $1 \mathrm{~kb}$ (Supplementary Figure 2a). Peaks were considered as gene-associated if the peak center was located between $1 \mathrm{~kb}$ upstream from the TSS (Transcription Start Site) and $1 \mathrm{~kb}$ downstream from the TTS (Transcription Terminal Site) of a gene. Of note, one feature of the Drosophila genome is that intergenic and intronic regions contain numerous simple repeat and transposons, as annotated by the dm6 version of Drosophila genome. 
Motif analysis. Two motif prediction methods, SeqPos in Cistrome $\mathrm{e}^{39}$ and MEME$\mathrm{ChIP}^{40}$, were used to obtain enriched motifs on $6 \mathrm{~mA}$ peak regions. Only sequences located on the central $200 \mathrm{bp}$ from peak summits were considered for motif analysis. We used the top 5,000 6mA peaks from 0.75 -h stage to predict the most enriched motifs by SeqPos and obtained the top 10 enriched motifs with the zscore $<-15$ and the highest hits. Then, total $0.75-\mathrm{h} 6 \mathrm{~mA}$ peaks were used to predict the most enriched motifs by MEME-ChIP, and obtained the top 10 candidates with $E$ value $<0.05$ and the highest hits. Motif hits were detected using Find Individual Motif Occurrences (FIMO) ${ }^{41}$ with a cutoff $\mathrm{p}$ value of $1 \mathrm{E}-4$.

RNA-seq data analysis. We performed RNA-seq for wild-type embryo samples at the $0.75-, 3-$, and 6-h developmental stages with 125 bp paired-end highthroughput sequencing by Illumina HiSeq 2500 V4 platform and RNA-seq for 3-h wild type, maternal jumu mutant, maternal and zygotic jumu mutant embryo samples with 150 bp paired-end high-throughput sequencing by NovaSeq platform. The FastQC software (http://www.bioinformatics.babraham.ac.uk/projects/fastqc/) was used to make sure that the sequencing data were high quality. Then, all the sequencing reads that aligned to ribosomal RNA (rRNA) sequences were removed by Bowtie $2^{34}$. The remaining reads were mapped to the December 2014 assembly of the D. melanogaster genome (UCSC version dm6, BDGP Release 6, unlocalized scaffolds excluded) using STAR ${ }^{42}$ with the default parameters. Differential expression analysis between the different developmental stages was carried out by cuffdiff from the cufflinks package ${ }^{43}$. FPKM (fragments per kilobase of transcript per million fragments mapped) was used to represent gene expression levels. Kmeans clustering of the differentially expressed genes in the three developmental stages was performed using Cluster 3.0 (clustering method: K-means clustering, Distance metric: Euclidean distance $)^{44}$.

To define genes significantly regulated by the maternal Jumu (GSRJ), we used jumu $u^{(\mathrm{M}-, \mathrm{Z}+)}$ and jumu $\mathrm{u}^{(\mathrm{M}-\mathrm{Z}-)}$ mutant embryos RNA-seq data sets at 3-h stage and selected genes with the same regulated direction in jumu $u^{(\mathrm{M}-, \mathrm{Z}+)}$ mutant and jum $u^{(\mathrm{M}-, \mathrm{Z}-)}$ mutant, compared with wild type, with cuffdiff $q$ value $<0.001$.

To define Zelda target genes, we generated the RNA-seq dataset of embryos (3-h stage) with overexpression of maternal Zelda. We integrated this dataset with the Zelda ChIP-seq data ${ }^{27}$ published previously to perform the Binding and Expression Target Analysis (BETA) ${ }^{45}$ analysis with parameters "-- df $0.05-\mathrm{c} 0.05$ ". And finally identified 3,165 Zelda target genes with FDR $<0.001$.

Transposon expression. $D M A D$ knockdown RNA-seq data sets of 6-h embryos were generated by paired-end with $125 \mathrm{bp}$ high-throughput sequencing using Illumina HiSeq 2500 platform V4 by Berry Genomics. The 6-h wild type and $D M A D$ knockdown RNA-seq data were aligned to the Drosophila genome (UCSC version dm6, BDGP Release 6, unlocalized scaffolds excluded) using STAR ${ }^{42}$ with the default parameters and "--outFilterMultimapNmax 100 --winAn-

chorMultimapNmax 100". The read counts of genes and transposons were calculated by featureCounts ${ }^{46}$ and the differential expression analysis was used by DESeq $2^{47}$.

Gene ontology (GO) analysis. The gene set was uploaded to the GO website (http://geneontology.org/) and PANTHER ${ }^{48}$ was used for GO enrichment analysis. The top 20 biological process terms for each group of genes were used to produce a heatmap with the R package pheatmap (https://CRAN.R-project.org/ package $=$ pheatmap).

Proposed regulatory network. The regulatory network of the Jumu target genes GSRJ and the target genes of Zelda was displayed using Cytoscape ${ }^{49}$ v3.6.1.

Statistical analysis. $P$ values were calculated by hypergeometric test or Student's $t$ test.

Reporting Summary. Further information on research design is available in the Nature Research Reporting Summary linked to this article.

\section{Data availability}

All sequencing data are available at GEO site under accession no. GSE86795. All Public data sets used in this study were downloaded from GEO. The source data underlying Figs. 2a-e, 3b-f, 4a, c-e, 5a, c, e-g, 6a, b and 7a and Supplementary Figures 1a-c, h, 2a-g, $3 b-c, 4 c, 5 a, c, d, f, g, 6 a, b, f$ and $7 \mathrm{a}-\mathrm{g}$ are provided as a Source Data file.

\section{Code availability}

The custom Perl and R scripts used in this study are available on request to the corresponding authors.

Received: 19 March 2019 Accepted: 19 April 2019

Published online: 17 May 2019

\section{References}

1. Cheng, X. Structure and function of DNA methyltransferases. Annu Rev. Biophys. Biomol. Struct. 24, 293-318 (1995).

2. Ratel, D., Ravanat, J. L., Berger, F. \& Wion, D. N6-methyladenine: the other methylated base of DNA. Bioessay 28, 309-315 (2006).

3. Smith, Z. D. \& Meissner, A. DNA methylation: roles in mammalian development. Nat. Rev. Genet 14, 204-220 (2013).

4. Feng, S., Jacobsen, S. E. \& Reik, W. Epigenetic reprogramming in plant and animal development. Science 330, 622-627 (2010).

5. Kriaucionis, S. \& Heintz, N. The nuclear DNA base 5-hydroxymethylcytosine is present in Purkinje neurons and the brain. Science 324, 929-930 (2009).

6. Tahiliani, M. et al. Conversion of 5-methylcytosine to 5hydroxymethylcytosine in mammalian DNA by MLL partner TET1. Science 324, 930-935 (2009).

7. Wion, D. \& Casadesus, J. N6-methyl-adenine: an epigenetic signal for DNAprotein interactions. Nat. Rev. Microbiol 4, 183-192 (2006).

8. Fu, Y. et al. N6-methyldeoxyadenosine marks active transcription start sites in chlamydomonas. Cell 161, 879-892 (2015).

9. Greer, E. L. et al. DNA methylation on N6-adenine in C. elegans. Cell 161, 868-878 (2015).

10. $\mathrm{Wu}, \mathrm{T}$. P. et al. DNA methylation on $\mathrm{N}$-adenine in mammalian embryonic stem cells. Nature 532, 329-333 (2016).

11. Zhang, G. et al. N6-methyladenine DNA modification in Drosophila. Cell 161, 893-906 (2015).

12. Koziol, M. J. et al. Identification of methylated deoxyadenosines in vertebrates reveals diversity in DNA modifications. Nat. Struct. Mol. Biol. 23, 24-30 (2016).

13. Yao, B. et al. Active N(6)-methyladenine demethylation by DMAD regulates gene expression by coordinating with polycomb protein in neurons. Mol. cell 71, 848-857 e846 (2018).

14. Iwafuchi-Doi, M. \& Zaret, K. S. Pioneer transcription factors in cell reprogramming. Genes Dev. 28, 2679-2692 (2014).

15. Drouin, J. Minireview: pioneer transcription factors in cell fate specification. Mol. Endocrinol. 28, 989-998 (2014).

16. Walser, C. B. \& Lipshitz, H. D. Transcript clearance during the maternal-tozygotic transition. Curr. Opin. Genet. Dev. 21, 431-443 (2011).

17. Tadros, W. \& Lipshitz, H. D. The maternal-to-zygotic transition: a play in two acts. Development 136, 3033-3042 (2009).

18. Lee, M. T., Bonneau, A. R. \& Giraldez, A. J. Zygotic genome activation during the maternal-to-zygotic transition. Annu Rev. Cell Dev. Biol. 30, 581-613 (2014).

19. Langley, A. R., Smith, J. C., Stemple, D. L. \& Harvey, S. A. New insights into the maternal to zygotic transition. Development 141, 3834-3841 (2014).

20. Liang, H. L. et al. The zinc-finger protein Zelda is a key activator of the early zygotic genome in Drosophila. Nature 456, 400-403 (2008).

21. Nien, C. Y. et al. Temporal coordination of gene networks by Zelda in the early Drosophila embryo. PLoS Genet. 7, e1002339 (2011).

22. Staudt, N., Fellert, S., Chung, H. R., Jackle, H. \& Vorbruggen, G. Mutations of the Drosophila zinc finger-encoding gene vielfaltig impair mitotic cell divisions and cause improper chromosome segregation. Mol. Biol. Cell 17, 2356-2365 (2006)

23. Schulz, K. N. et al. Zelda is differentially required for chromatin accessibility, transcription factor binding, and gene expression in the early Drosophila embryo. Genome Res. 25, 1715-1726 (2015).

24. Nunes da Fonseca, R. \& Venancio, T. M. Maternal or zygotic: unveiling the secrets of the Pancrustacea transcription factor zelda. PLoS Genet. 14, e1007201 (2018).

25. Li, X. Y., Harrison, M. M., Villalta, J. E., Kaplan, T. \& Eisen, M. B. Establishment of regions of genomic activity during the Drosophila maternal to zygotic transition. eLife 3, 03737 (2014).

26. Tang, A. H., Neufeld, T. P., Rubin, G. M. \& Muller, H. A. Transcriptional regulation of cytoskeletal functions and segmentation by a novel maternal pair-rule gene, lilliputian. Development 128, 801-813 (2001).

27. Harrison, M. M., Li, X. Y., Kaplan, T., Botchan, M. R. \& Eisen, M. B. Zelda binding in the early Drosophila melanogaster embryo marks regions subsequently activated at the maternal-to-zygotic transition. PLoS Genet. 7, e1002266 (2011).

28. Fu, S., Nien, C. Y., Liang, H. L. \& Rushlow, C. Co-activation of microRNAs by Zelda is essential for early Drosophila development. Development 141, 2108-2118 (2014)

29. Katoh, M., Igarashi, M., Fukuda, H., Nakagama, H. \& Katoh, M. Cancer genetics and genomics of human FOX family genes. Cancer Lett. 328, 198-206 (2013).

30. Li, Z., Tuteja, G., Schug, J. \& Kaestner, K. H. Foxa1 and Foxa2 are essential for sexual dimorphism in liver cancer. Cell 148, 72-83 (2012).

31. Williams, K. et al. TET1 and hydroxymethylcytosine in transcription and DNA methylation fidelity. Nature 473, 343-348 (2011). 
32. Rasmussen, K. D. et al. Loss of TET2 in hematopoietic cells leads to DNA hypermethylation of active enhancers and induction of leukemogenesis. Genes \&. development 29, 910-922 (2015).

33. Apweiler, R. et al. UniProt: the Universal Protein knowledgebase. Nucleic acids Res. 32, D115-D119 (2004).

34. Langmead, B. \& Salzberg, S. L. Fast gapped-read alignment with Bowtie 2. Nat. methods 9, 357-359 (2012).

35. Zhang, Y. et al. Model-based analysis of ChIP-Seq (MACS). Genome Biol. 9, R137 (2008).

36. Shin, H., Liu, T., Manrai, A. K. \& Liu, X. S. CEAS: cis-regulatory element annotation system. Bioinformatics 25, 2605-2606 (2009).

37. Thorvaldsdottir, H., Robinson, J. T. \& Mesirov, J. P. Integrative Genomics Viewer (IGV): high-performance genomics data visualization and exploration. Brief. Bioinforma. 14, 178-192 (2013).

38. Heinz, S. et al. Simple combinations of lineage-determining transcription factors prime cis-regulatory elements required for macrophage and B cell identities. Mol. cell 38, 576-589 (2010).

39. Liu, T. et al. Cistrome: an integrative platform for transcriptional regulation studies. Genome Biol. 12, R83 (2011).

40. Machanick, P. \& Bailey, T. L. MEME-ChIP: motif analysis of large DNA datasets. Bioinformatics 27, 1696-1697 (2011).

41. Grant, C. E., Bailey, T. L. \& Noble, W. S. FIMO: scanning for occurrences of a given motif. Bioinformatics 27, 1017-1018 (2011).

42. Dobin, A et al. STAR: ultrafast universal RNA-seq aligner Bioinformatics. 15-21 (2013).

43. Trapnell, C. et al. Transcript assembly and quantification by RNA-Seq reveals unannotated transcripts and isoform switching during cell differentiation. Nat. Biotechnol. 28, 511-515 (2010).

44. Eisen, M. B., Spellman, P. T., Brown, P. O. \& Botstein, D. Cluster analysis and display of genome-wide expression patterns. Proc. Natl Acad. Sci. USA 95, 14863-14868 (1998)

45. Wang, S. et al. Target analysis by integration of transcriptome and ChIP-seq data with BETA. Nat. Protoc. 8, 2502-2515 (2013).

46. Liao, Y., Smyth, G. K. \& Shi, W. featureCounts: an efficient general purpose program for assigning sequence reads to genomic features. Bioinformatics 30, 923-930 (2014).

47. Love, M. I., Huber, W. \& Anders, S. Moderated estimation of fold change and dispersion for RNA-seq data with DESeq2. Genome Biol. 15, 550 (2014).

48. Mi, H., Muruganujan, A., Casagrande, J. T. \& Thomas, P. D. Large-scale gene function analysis with the PANTHER classification system. Nat. Protoc. 8, 1551-1566 (2013).

49. Shannon, P. et al. Cytoscape: a software environment for integrated models of biomolecular interaction networks. Genome Res. 13, 2498-2504 (2003).

\section{Acknowledgements}

The authors thank Dr. Shirley Liu for her critical comments and Yuanyuan Chen and Zhenwei Yang at Core Facility for Protein Research (Institute of Biophysics, CAS) for technical help with Biacore (SPR) experiments. This work was supported by the Ministry of Science and Technology of China (2016YFA0100400, 2018YFC1003300), Strategic Priority Research Program of the Chinese Academy of Sciences (XDB13000000, XDB1900000), Natural Science Foundation of China (31590831, 31671498, 31871294), National Key R\&D Program of China (2017YFA0506800, 2016YFC0901700) and CAS Interdisciplinary Innovation Team. Data analysis and computing resource was supported by Center of Big Data Research in Health (http://bigdata.ibp.ac.cn), Institute of Biophysics, Chinese Academy of Sciences.

\section{Author contributions}

S.H., G.Z., Y.T., Q.S., and D.C. designed the experiments; G.Z., Y.G., R.S., Z. Cao, Z. Chen, X.Z., Y.L., X.W., and W.Z. performed experiments; S.H., J.W., J.Y., P.Z., Y.Z., C.H., Y.T., Q.S., and D.C. analyzed data; S.H., G.Z., J.W., Y.T., Q.S., and D.C. wrote the paper. All authors provided intellectual input, vetted and approved the final manuscript.

\section{Additional information}

Supplementary Information accompanies this paper at https://doi.org/10.1038/s41467019-10202-3.

Competing interests: The authors declare no competing interests.

Reprints and permission information is available online at http://npg.nature.com/ reprintsandpermissions/

Journal peer review information: Nature Communications thanks the anonymous reviewer(s) for their contribution to the peer review of this work. Peer reviewer reports are available

Publisher's note: Springer Nature remains neutral with regard to jurisdictional claims in published maps and institutional affiliations.

Open Access This article is licensed under a Creative Commons Attribution 4.0 International License, which permits use, sharing, adaptation, distribution and reproduction in any medium or format, as long as you give appropriate credit to the original author(s) and the source, provide a link to the Creative Commons license, and indicate if changes were made. The images or other third party material in this article are included in the article's Creative Commons license, unless indicated otherwise in a credit line to the material. If material is not included in the article's Creative Commons license and your intended use is not permitted by statutory regulation or exceeds the permitted use, you will need to obtain permission directly from the copyright holder. To view a copy of this license, visit http://creativecommons.org/ licenses/by/4.0/.

(C) The Author(s) 2019 